\title{
Conserved charge of a gravity theory with $p$-form gauge fields and its property under Kaluza-Klein reduction
}

\author{
Jun-Jin Peng* \\ School of Physics and Electronic Science, Guizhou Normal University, \\ Guiyang, Guizhou 550001, People's Republic of China
}

\begin{abstract}
In this paper, we investigate the conserved charges of generally diffeomorphism invariant gravity theories with a wide variety of matter fields, particularly of the theories with multiple scalar fields and $p$-form potentials, in the context of the off-shell generalized Abbott-Deser-Tekin (ADT) formalism. We first construct a new off-shell ADT current that consists of the terms for the variation of a Killing vector and expressions of the field equations as well as the Lie derivative of a surface term with respect to the Killing vector within the framework of generally diffeomorphism invariant gravity theories involving various matter fields. After deriving the off-shell ADT potential corresponding to this current, we propose a formula of conserved charges for these theories. Next, we derive the off-shell ADT potential associated with the generic Lagrangian that describes a large range of gravity theories with a number of scalar fields and $p$ form potentials. Finally, the properties of the off-shell generalized ADT charges for the theory of Einstein gravity and the gravity theories with a single $p$-form potential are investigated by performing Kaluza-Klein dimensional reduction along a compactified direction. The results indicate that the charge contributed by all the fields in the lowerdimensional theory is equal to that of the higher-dimensional one at mathematical level with the hypothesis that the higher-dimensional spacetime allows for the existence of the compactified dimension. In order to illustrate our calculations, the mass and angular momentum for the five-dimensional rotating Kaluza-Klein black holes are explicitly evaluated as an example.
\end{abstract}

*pengjjph@163.com 


\section{Contents}

1 Introduction 2

2 The off-shell generalized ADT currents and conserved charges of diffeomorphism invariant gravity theories with arbitrary matter fields

3 Conserved charges of gravity theories with scalar fields and $p$-form potentials

4 Conserved charges of (D+1)-dimensional Einstein gravity under KaluzaKlein reduction

5 Properties for conserved charges in gravity theories with n-form field strength under Kaluza-Klein reduction

6 An example: conserved charges of Kaluza-Klein black holes

7 Conclusions and discussions

A The off-shell Noether currents and potentials of the Lagrangians $\mathcal{L}_{M}$ and $\mathcal{L}_{\phi}$

B The (D+1)-dimensional Christoffel symbols and surface term

D The Kaluza-Klein reduction of the Lagrangian with $p$-form potentials

E The $\hat{K}_{(\hat{F})}^{\mu \nu}$ potential in terms of the D-dimensional fields

\section{Introduction}

The definition of conserved charges in various gravity theories is of great importance for the understanding of many physics properties of spacetime. As is well known, its remarkable and 
successful applications are those in spacetime thermodynamics and black hole physics. Due to this, much work has been devoted to seeking feasible approaches to define the conserved charges of gravity theories appropriately. A rather effective route to do this is to make use of Noether theorem.

Till now several approaches in terms of the Noether procedure have been proposed to compute the conserved charges. One of them is the Abbott-Deser-Tekin (ADT) method [1, 2, 3]. The ADT formalism, which is defined in terms of the Noether potential got through the linearized perturbation for the expression of equation of motion in a fixed background of (A)dS spacetime, has made some progress on computation scheme for conserved charges of asymptotically (A)dS black holes in various gravity theories. Since the background metric is a vacuum solution of the field equation, the Noether potential in the original ADT formalism is on-shell. One of the applications for the ADT formalism is to compute the conserved charges within the context of three-dimensional topologically massive gravity in the work [4, which was subsequently extended to nonasymptotically AdS black holes in the same thoery in [5]. Notably, in the latter, although the current is on-shell, the potential was extracted from the expression of the current derived without the requirement that the background metric is on-shell. This form of the current allows for the possibility of the construction of an off-shell current in the context of pure gravity theories. Afterwards, the development for the derivation of the potential was extended to compute the mass and angular momentum of three-dimensional Chern-Simons black holes [6, 8] and black holes in three-dimensional new massive gravity [7].

Recently, in Ref. [9], relieving the constraint that the background metric satisfies the field equations, Kim, Kulkarni and Yi proposed a quasi-local formulation of conserved charges within the framework of generic covariant pure gravity theories by constructing an off-shell ADT current to generalize the conventional on-shell Noether potential in the original ADT formalism to off-shell level, as well as following works [27, 28, 29] to incorporate a single parameter path in the space of solutions into their definition. These modifications make it more operable to evaluate the Noether potential in terms of the corresponding current and the procedure of computation become more convenient to manipulate. Owing to these, the off-shell generalized formalism for the quasi-local conserved charges provides another fruitful way to evaluate the ADT charges for various theories of gravity and it has been extended to investigate the conserved charges of a wide scope of gravity theories with or without matter fields [10, 11, 12, 13, 14, 15, 16, 17, 18, 19, 20, 21]. Particularly, in [10], 
the method in [9] was generalized to the gravity theories in the presence of matter fields.

In the work [11, reconsidering the conserved charges of generally diffeomorphism invariant gravity theories with only gravitational field, we constructed an off-shell generalized ADT current that takes a different form from the one given in [9] through the linear combination of the variation of the Bianchi identity for the expression of the field equation and the Lie derivative for the variation of the Lagrangian along a Killing vector. However, both the currents are equivalent since the Lie derivative of a surface term with respect to the Killing vector disappears. Our procedure to construct the off-shell ADT current is rather simple and the current naturally yields its corresponding potential that completely coincides with the one in [9]. A question naturally arises, i.e. whether the procedure in [11] is applicable to construct the off-shell ADT current and potential of diffeomorphism invariant gravity theories in the presence of a wide variety of matter fields.

On the other hand, $p$-form gauge fields appear systematically within the context of the string theories, the higher-dimensional supergravity theories as well as the brane theories, for example, in the ten-dimensional type IIB and eleven-dimensional supergravity theories. They have attracted much attention in recent years. In particular, some exact solutions in the supergravity theories have been found. For several of them see the references [38, 39, 40, 41, 42, 43. To understand the behaviour of the $p$-form gauge fields on the conserved charges for gravity theories with such fields, it is of great interest to apply the off-shell generalized ADT formalism to fully identify their contribution. If we do this, the outcome will be able to provide a basis for further investigation on thermodynamics and other related properties of the solutions found in these theories. Besides, as is well-known, if the higher-dimensional spacetime allows for the existence of a compactified dimension, by performing Kaluza-Klein dimensional reduction along this compactified direction, the higher-dimensional gravity theories can be described by reduced gravity theories involving a series of lower-dimensional fields, such as the gravitational field, the Kaluza-Klein vector, the dilaton field and so on. Through such a procedure, a natural question is how the conserved charges associated with the higher-dimensional theories of gravity to behave in the lower-dimensional reduced theories after the dimensional reduction. We wonder wether a consistent Kaluza-Klein dimensional reduction can yield consistent conserved charges of the gravity theories.

In consideration of the above issues, the main motivation of the present work is to shed some light on the conserved charges of generally diffeomorphism invariant gravity theories 
with various matter fields, particularly of the theories with a number of scalar fields and $p$-form potentials, in the context of the off-shell generalized ADT formalism proposed in [9, 10]. To achieve this, along the line of the work [11, the off-shell ADT formalism is extended to these gravity theories by constructing a new generic off-shell ADT current without the condition that the Killing vector associated with the conserved charge has to be fixed. In terms of the off-shell ADT potential constructed from this current, the formula for the conserved charges is presented. In particular, we propose a very general Lagrangian that describes a large range of gravity theories with scalar fields and $p$-form potentials. Accordingly, the conserved charges for this Lagrangian are explicitly investigated. What is more, another motivation is to gain a good understanding of the properties for the conserved charges under the Kaluza-Klein dimensional reduction. This is achieved by exploiting the off-shell ADT charges of $(D+1)$-dimensional pure Einstein gravity and gravity theories with a single $p$-form gauge field before and after performing Kaluza-Klein reduction on an $S^{1}$ circle. The results demonstrate that the off-shell ADT charges exhibit the property of invariance under the dimensional reduction.

The remainder of this paper is organized as follows: In section 2, we derive the general formalism for conserved charges of diffeomorphism covariant gravity theories with arbitrary matter fields by constructing the off-shell generalized ADT currents and potentials for such theories in a new manner. In section 3, we move on to apply the general formalism of conserved charges in the previous section to generic covariant gravity theories in the presence of multiple scalar fields and $p$-form potentials. In section 4 , we study the properties of conserved charges of $(D+1)$-dimensional Einstein gravity by performing Kaluza-Klein reduction on a circle. In section 5, the analysis on Einstein gravity is extended to investigate the behaviour of conserved charges within the framework of gravity theories with multiple scalar fields and a single ( $n-1)$-form gauge field. In order to illustrate our calculations, we consider to explicitly compute the conserved charges of five-dimensional rotating KaluzaKlein black holes in light of the off-shell generalized ADT formalism within section 6, The last section is our conclusions and discussions on future work. To gain more details on our derivations in the main body of the text, several appendixes are presented. Particularly, in Appendix $\mathbf{A}$, the off-shell Noether currents and potentials for the Lagrangians with multiple scalar fields and $p$-form potentials are calculated in details. The Appendix $\mathbf{D}$ is devoted to the discussion of Kaluza-Klein dimensional reduction to the generic Lagrangian that consists of a number of scalar fields, multiple $p$-form potentials, as well as a gravitational field, 
along a compactified direction.

\section{The off-shell generalized ADT currents and conserved charges of diffeomorphism invariant gravity theories with arbitrary matter fields}

In our previous work [11, providing a new route varying the Bianchi identity for the expression of the field equations, together with help of the Killing equation for a Killing vector that generates a spacetime symmetry, we got another form for the off-shell generalized ADT current of generally diffeomorphism covariant gravity theories with only gravitational field given in [9]. The new form of the current contains an additional term that is just one half of the Lie derivative of a surface term with respect to the Killing vector. However, in essence, this current makes no difference to the one presented in [9] because of the vanishing of the Lie derivative for the surface term.

In this section, we generalize the derivation in the work [11 to gravity theories with a wide variety of matter fields, satisfying the criteria of general covariance. As a result, we propose a generalized off-shell ADT current that arises from the diffeomorphism symmetry of the Lagrangian and the vanishing of the divergence for the contraction between the Killing vector and a generally non-symmetric 2-rank tensor, which is always made up of expressions of the equations of motion for gravitational field and matter fields. The newly improved current, including the Lie derivative of the surface term with respect to the Killing vector and extra terms involving the variation of the Killing vector in comparison with the one given in [11, differs from the current proposed in [10], where the off-shell generalized ADT formalism for the pure gravity theories in the work [9] was extended to study conserved charges of covariant gravity theories in the presence of matter fields. Moving on to construct out the off-shell ADT potential from the current, we further present a formula for the conserved charges of these gravity theories along the line of the work [9].

We proceed by considering the Lagrangian describing $D$-dimensional generally diffeomorphism covariant gravity theories with various matter fields

$$
\mathcal{L}=\sqrt{-g} L\left(g_{\mu \nu}, \psi^{(r)}\right),
$$

in which the quantity $\psi^{(r)}$ is taken to run over all the matter fields of the theories. The variation of the general Lagrangian (2.1) with respect to the gravitational field and all the 
matter fields reads

$$
\delta \mathcal{L}=\sqrt{-g}\left(\mathcal{E}_{\mu \nu}^{(g r)} \delta g^{\mu \nu}+\sum_{r} \mathcal{E}_{\psi^{(r)}} \delta \psi^{(r)}+\nabla_{\mu} \Theta^{\mu}\left(\delta g, \delta \psi^{(r)}\right)\right)
$$

where $\mathcal{E}_{\mu \nu}^{(g r)}$ and $\mathcal{E}_{\psi^{(r)}}$ are the expressions for the field equations, and $\Theta^{\mu}\left(\delta g, \delta \psi^{(r)}\right)$ is a surface term. When the variation of the gravitation field $g_{\mu \nu}$ and the matter fields $\psi^{(r)}$ behaves as their Lie derivative along any smooth vector field $\zeta^{\mu}$, namely, $\delta g_{\mu \nu} \rightarrow \mathcal{L}_{\zeta} g_{\mu \nu}$ and $\delta \psi^{(r)} \rightarrow \mathcal{L}_{\zeta} \psi^{(r)}$ under diffeomorphisms $x^{\mu} \rightarrow x^{\mu}-\zeta^{\mu}$ generated by the vector field $\zeta^{\mu}$, it was shown in [10, 26] that the following identity

$$
2 \nabla_{\mu}\left(\mathcal{E}^{\mu \nu} \zeta_{\nu}\right)=\mathcal{E}_{(g r)}^{\mu \nu} \mathcal{L}_{\zeta} g_{\mu \nu}-\sum_{r} \mathcal{E}_{\psi^{(r)}} \mathcal{L}_{\zeta} \psi^{(r)}
$$

generally holds for diffeomorphism invariant gravity theories. The identity (2.3) plays a key role for deriving the off-shell Noether current. Its left-hand side is the divergence for the contraction of the vector $\zeta_{\nu}$ with the 2-rank tensor $\mathcal{E}^{\mu \nu}$, which is in general unnecessary to be symmetric and is defined by

$$
\mathcal{E}^{\mu \nu}=\mathcal{E}_{(g r)}^{\mu \nu}-\frac{1}{2} Z_{(\psi)}^{\mu \nu},
$$

where the 2-rank tensor $Z_{(\psi)}^{\mu \nu}$ is generally proportional to some combination of the expressions of the field equations $\mathcal{E}_{\psi^{(r)}}$ and it vanishes when $\mathcal{E}_{\psi^{(r)}}=0$. Particularly, in the absence of matter fields, Eq. (2.3) yields $\nabla_{\mu} \mathcal{E}_{(g r)}^{\mu \nu}=0$, which is just the Bianchi identity for covariant gravity theories with only gravitational field [11. Replacing the variation in Eq. (2.2) by the Lie derivative along the vector field $\zeta^{\mu}$, as well as making use of Eq. (2.3), we obtain the following conservation law

$$
\nabla_{\mu} J^{\mu}=0
$$

where the divergence-free vector $J^{\mu}$, defined by

$$
J^{\mu}=2 \mathcal{E}^{\mu \nu} \zeta_{\nu}+\zeta^{\mu} L-\Theta^{\mu}\left(\mathcal{L}_{\zeta} g, \mathcal{L}_{\zeta} \psi^{(r)}\right),
$$

is an off-shell Noether current [9, 10, 22] in the sense that the conservation law for this Noether current holds without the assumption that the equations of motion for the fields are valid. Furthermore, the off-shell Noether potential $K^{\mu \nu}$ corresponding to the current can be defined as

$$
J^{\mu}=\nabla_{\nu} K^{\mu \nu}
$$


Next, we focus our attention on detailed derivation of the off-shell generalized ADT current and its corresponding potential on basis of the above off-shell Noether current and potential. In the case where the vector $\zeta^{\mu}$ is replaced by a Killing vector $\xi^{\mu}$ that satisfies the following symmetry conditions:

$$
\begin{aligned}
\mathcal{L}_{\xi} g_{\mu \nu} & =\nabla_{\mu} \xi_{\nu}+\nabla_{\nu} \xi_{\mu}=0, \\
\mathcal{L}_{\xi} \psi^{(r)} & =0
\end{aligned}
$$

the right-hand side of Eq. (2.3) vanishes, which directly gives rise to

$$
\nabla_{\mu}\left(\mathcal{E}^{\mu \nu} \xi_{\nu}\right)=0
$$

i.e. the divergence for the contraction of the $\mathcal{E}^{\mu \nu}$ tensor and the Killing vector $\xi_{\nu}$ takes zero value. This can be regarded as a consequence of the diffeomorphism and symmetry of matter fields. Significantly, one will see that the identity (2.9) plays a vital role in constructing the off-shell generalized ADT current in the following. Varying this identity, we get

$$
\delta\left[\nabla_{\mu}\left(\mathcal{E}^{\mu \nu} \xi_{\nu}\right)\right]=\nabla_{\mu} \mathcal{J}_{[1]}^{\mu}=0
$$

where the current

$$
\mathcal{J}_{[1]}^{\mu}=\delta \mathcal{E}^{\mu \nu} \xi_{\nu}+\mathcal{E}^{\mu \nu} \xi^{\lambda} \delta g_{\nu \lambda}+\frac{1}{2} \mathcal{E}^{\mu \nu} \xi_{\nu} g^{\rho \sigma} \delta g_{\rho \sigma}+\mathcal{E}_{\nu}^{\mu} \delta \xi^{\nu}
$$

Unlike in [9, 10, 11, here and in what follows we do not impose the constraint of the vanishing of the variation for the Killing vector $\xi^{\mu}$, i.e., $\delta \xi^{\mu}=0$, on any current, although this generally holds for the Killing vectors associated with energy and angular momentum. In particular, when all the fields satisfy the equations of motion, namely, $\mathcal{E}_{(g r)}^{\mu \nu}=0=\mathcal{E}_{\psi^{(r)}}$, the current $\mathcal{J}_{[1]}^{\mu}$ becomes the original on-shell ADT current [1, 2].

On the other hand, letting the Lie derivative with respect to the Killing vector $\xi^{\mu}$ act on the variation equation (2.2) of the Lagrangian, we have

$$
\begin{aligned}
\mathcal{L}_{\xi} \delta(\sqrt{-g} L) & =\sqrt{-g} \nabla_{\mu}\left[\xi^{\mu} \mathcal{E}_{\rho \sigma}^{(g r)} \delta g^{\rho \sigma}+\xi^{\mu} \sum_{r} \mathcal{E}_{\psi^{(r)}} \delta \psi^{(r)}+\mathcal{L}_{\xi} \Theta^{\mu}\left(\delta g, \delta \psi^{(r)}\right)\right] \\
& =-\sqrt{-g} \nabla_{\mu}\left(L \delta \xi^{\mu}\right)
\end{aligned}
$$

which yields a conserved current that reads

$$
\mathcal{J}_{[2]}^{\mu}=\xi^{\mu} \mathcal{E}_{\rho \sigma}^{(g r)} \delta g^{\rho \sigma}+\xi^{\mu} \sum_{r} \mathcal{E}_{\psi^{(r)}} \delta \psi^{(r)}+\mathcal{L}_{\xi} \Theta^{\mu}\left(\delta g, \delta \psi^{(r)}\right)+L \delta \xi^{\mu}
$$


This current, together with the $\mathcal{J}_{[1]}^{\mu}$ current in Eq. (2.11), should be able to underlie the construction of a new Noether current. In fact, through a linear combination of the currents $\mathcal{J}_{[1]}^{\mu}$ and $\mathcal{J}_{[2]}^{\mu}$, a new off-shell generalized ADT current can be proposed as

$$
\begin{aligned}
\mathcal{J}_{A D T}^{\mu}= & \mathcal{J}_{[1]}^{\mu}+\frac{1}{2} \mathcal{J}_{[2]}^{\mu} \\
= & \delta \mathcal{E}^{\mu \nu} \xi_{\nu}+\mathcal{E}^{\mu \nu} \xi^{\lambda} \delta g_{\nu \lambda}+\frac{1}{2} \mathcal{E}^{\mu \nu} \xi_{\nu} g^{\rho \sigma} \delta g_{\rho \sigma}+\frac{1}{2} \xi^{\mu} \mathcal{E}_{\rho \sigma}^{(g r)} \delta g^{\rho \sigma} \\
& +\frac{1}{2} \xi^{\mu} \sum_{r} \mathcal{E}_{\psi^{(r)}} \delta \psi^{(r)}+\frac{1}{2} \mathcal{L}_{\xi} \Theta^{\mu}\left(\delta g, \delta \psi^{(r)}\right) \\
& +\frac{1}{2} L \delta \xi^{\mu}+\mathcal{E}^{\mu}{ }_{\nu} \delta \xi^{\nu},
\end{aligned}
$$

where the current $\mathcal{J}_{A D T}^{\mu}$ can be thought of as a generalization of the off-shell ADT current for pure gravity theories in [11] to the one for covariant gravity theories with arbitrary matter fields when the variation of the Killing vector $\delta \xi^{\mu}$ vanishes, namely, $\delta \xi^{\mu}=0$. In addition, in comparison with the off-shell ADT current for the gravity theories with matter fields in [10], the current in Eq. (2.14), containing the term $\mathcal{L}_{\xi} \Theta^{\mu}\left(\delta g, \delta \psi^{(r)}\right)$ as well as the terms with the variation of the Killing vector, gives rise to a different form from the one given in [10]. However, both of the currents are essentially equivalent since one can verify that $\mathcal{L}_{\xi} \Theta^{\mu}\left(\delta g, \delta \psi^{(r)}\right)=0$ for the generally diffeomorphism covariant Lagrangian (2.1) under the condition that the variation of the $\xi^{\mu}$ Killing vector, obeying Eq. (2.8), disappears.

Our procedure to bring in the off-shell ADT current $\mathcal{J}_{A D T}^{\mu}$ is very simple since it is only constructed out of a combination for two generally satisfied equations, that is, the variation equation (2.10) of a divergence-free term made up of expressions of the field equations and a Killing vector, together with the equation (2.12) for the Lie derivative of the variation of the Lagrangian along the Killing vector. It is also unnecessary for us to fix the Killing vector in order to get the off-shell ADT current and the latter potential. What is more, we shall see that our formulation for the off-shell ADT current in turn makes a natural and practical construction for derivation of its corresponding potential in the following.

In terms of the off-shell ADT current $\mathcal{J}_{A D T}^{\mu}$ given by Eq. (2.14), we go on to derive its corresponding potential $\mathcal{Q}_{A D T}^{\mu \nu}$, which is associated with the current through the well-known relation

$$
\mathcal{J}_{A D T}^{\mu}=\nabla_{\nu} \mathcal{Q}_{A D T}^{\mu \nu}
$$


To do this, the $\mathcal{J}_{A D T}^{\mu}$ current multiplied by the factor $\sqrt{-g}$ is expressed as

$$
\begin{aligned}
\sqrt{-g} \mathcal{J}_{A D T}^{\mu}= & \delta\left(\sqrt{-g} \mathcal{E}^{\mu \nu} \xi_{\nu}\right)+\frac{1}{2} \sqrt{-g} \xi^{\mu} \mathcal{E}_{\rho \sigma}^{(g r)} \delta g^{\rho \sigma}+\frac{1}{2} \sqrt{-g} \xi^{\mu} \sum_{r} \mathcal{E}_{\psi^{(r)}} \delta \psi^{(r)} \\
& +\frac{1}{2} \sqrt{-g}\left(\mathcal{L}_{\xi} \Theta^{\mu}\left(\delta g, \delta \psi^{(r)}\right)+L \delta \xi^{\mu}\right) .
\end{aligned}
$$

According to Eq. (2.6), the off-shell Noether current associated with the Killing vector $\xi^{\mu}$ is given by $J_{\xi}^{\mu}=J^{\mu}(\zeta \rightarrow \xi)$. Like in [23, 11], the $J_{\xi}^{\mu}$ current can also be defined as the equivalent form, i.e. $J_{\xi}^{\mu}=2 \mathcal{E}^{\mu \nu} \xi_{\nu}+\xi^{\mu} L$, stemming from that the disappearance of the Lie derivative for the gravitational field and all the matter fields with respect to the Killing vector leads to the vanishing of the $\Theta^{\mu}\left(\mathcal{L}_{\xi} g, \mathcal{L}_{\xi} \psi^{(r)}\right)$ term for a generally diffeomorphism invariant theory. Substituting the off-shell Noether current $J_{\xi}^{\mu}$ and the Lie derivative of the surface term $\Theta^{\mu}\left(\delta g, \delta \psi^{(r)}\right)$ given by

$$
\mathcal{L}_{\xi} \Theta^{\mu}\left(\delta g, \delta \psi^{(r)}\right)=-2 \nabla_{\nu}\left(\xi^{[\mu} \Theta^{\nu]}\left(\delta g, \delta \psi^{(r)}\right)\right)+\xi^{\mu} \nabla_{\nu} \Theta^{\nu}\left(\delta g, \delta \psi^{(r)}\right)
$$

into Eq. (2.16), we reexpress the current $\mathcal{J}_{A D T}^{\mu}$ as the form

$$
\begin{aligned}
\sqrt{-g} \mathcal{J}_{A D T}^{\mu}= & \frac{1}{2} \delta\left(\sqrt{-g} J_{\xi}^{\mu}\right)-\sqrt{-g} \nabla_{\nu}\left(\xi^{[\mu} \Theta^{\nu]}\left(\delta g, \delta \psi^{(r)}\right)\right) \\
& +\frac{1}{2} \delta\left[\sqrt{-g} \Theta^{\mu}\left(\mathcal{L}_{\xi} g, \mathcal{L}_{\xi} \psi^{(r)}\right)\right] .
\end{aligned}
$$

Due to Eq. (2.18), one can observe that the preservation of the $\mathcal{L}_{\xi} \Theta^{\mu}\left(\delta g, \delta \psi^{(r)}\right)$ term makes it rather natural lead the $\xi^{[\mu} \Theta^{\nu]}\left(\delta g, \delta \psi^{(r)}\right)$ term into the potential. Note that the surface term $\Theta^{\mu}\left(\mathcal{L}_{\xi} g, \mathcal{L}_{\xi} \psi^{(r)}\right)=0$ for the generally diffeomorphism covariant Lagrangian (2.1). We further send the current $\mathcal{J}_{A D T}^{\mu}$ into the form

$$
\begin{aligned}
\sqrt{-g} \mathcal{J}_{A D T}^{\mu} & =\partial_{\nu}\left[\frac{1}{2} \delta\left(\sqrt{-g} K_{\xi}^{\mu \nu}\right)-\sqrt{-g} \xi^{[\mu} \Theta^{\nu]}\left(\delta g, \delta \psi^{(r)}\right)\right] \\
& =\partial_{\nu}\left(\sqrt{-g} \mathcal{Q}_{A D T}^{\mu \nu}\right)
\end{aligned}
$$

where $K_{\xi}^{\mu \nu}=K^{\mu \nu}(\zeta \rightarrow \xi)$ is determined by Eq. (2.7). From Eq. (2.19), we draw out the important relationship between the generalized ADT potential $\mathcal{Q}_{A D T}^{\mu \nu}$ and the off-shell Noether potential $K_{\xi}^{\mu \nu}$, that is,

$$
\sqrt{-g} \mathcal{Q}_{A D T}^{\mu \nu}=\frac{1}{2} \delta\left(\sqrt{-g} K_{\xi}^{\mu \nu}\right)-\sqrt{-g} \xi^{[\mu} \Theta^{\nu]}\left(\delta g, \delta \psi^{(r)}\right) .
$$

Equivalently, one can define the off-shell generalized ADT potential as follows:

$$
\mathcal{Q}_{A D T}^{\mu \nu}=\frac{1}{2} \delta K_{\xi}^{\mu \nu}+\frac{1}{4} K_{\xi}^{\mu \nu} g^{\rho \sigma} \delta g_{\rho \sigma}-\xi^{[\mu} \Theta^{\nu]}\left(\delta g, \delta \psi^{(r)}\right) .
$$


Compared with the off-shell ADT potential given in [9, 10, 11, it is worth noting that the Killing vector $\xi^{\mu}$ involved in the potential (2.21) is unnecessary to be fixed when varying the potential $K_{\xi}^{\mu \nu}$, although they take the same forms. As it was explicitly demonstrated in [10, the off-shell ADT potential (2.21) is equivalent with the on-shell Noether potential derived via the well-known covariant phase space approach, proposed by Lee, Iyer and Wald (LIW) [24, 25, 26], despite the currents are different from each other. What is more, the ADT potential is consistent with the one defined via the Barnich-Brandt-Compere (BBC) method [27, 28, 29, 30], which developed the covariant phase space method. Due to these, the off-shell ADT formalism can be regarded as an equivalent generalization of the LIW method or the BBC method. Unlike in [9, 10, note that both the LIW and BBC approaches also do not impose the constraint that the variation of the Killing vector associated with the potential has to vanish as in the present work.

Furthermore, as it has been shown that the off-shell ADT current (2.14) arises from the linear combination of the current $\mathcal{J}_{[1]}^{\mu}$ and one half of the current $\mathcal{J}_{[2]}^{\mu}$, a new current in terms of another linear combination of both the two currents can be proposed as

$$
\check{\mathcal{J}}^{\mu}=\mathcal{J}_{[1]}^{\mu}+\frac{1}{2}(2 k+1) \mathcal{J}_{[2]}^{\mu},
$$

where the constant $k$ takes a generic value. A new potential corresponding to the current $\check{\mathcal{J}}^{\mu}$ is defined by

$$
\check{\mathcal{Q}}^{\mu \nu}=\mathcal{Q}_{A D T}^{\mu \nu}+k \mathcal{K}_{[2]}^{\mu \nu},
$$

where the Noether potential $\mathcal{K}_{[2]}^{\mu \nu}$ is determined by the equation $\mathcal{J}_{[2]}^{\mu}=\nabla_{\nu} \mathcal{K}_{[2]}^{\mu \nu}$. According to Eq. (2.23), one observes that $\check{\mathcal{Q}}^{\mu \nu}$ returns to the off-shell ADT potential $\mathcal{Q}_{A D T}^{\mu \nu}$ when $k=0$ and both the potentials $\check{\mathcal{Q}}^{\mu \nu}$ and $\mathcal{Q}_{A D T}^{\mu \nu}$ coincide with the original on-shell ADT potential when all the fields satisfy the equations of motion accompanying with the vanishing of the variation for the Killing vector.

Like in [9], by following the BBC approach [27, 28, 29, 30] to incorporate a single parameter path characterized by a parameter $s$, where $s \in[0,1]$, in the space of solutions, we define the covariant formulation of conserved charges associated with the off-shell ADT potential $\mathcal{Q}_{A D T}^{\mu \nu}$ in Eq. (2.20) by

$$
\mathcal{Q}=\frac{1}{8 \pi G_{(D)}} \int_{0}^{1} d s \int d \Sigma_{\mu \nu} \mathcal{Q}_{A D T}^{\mu \nu}\left(g, \psi^{(r)} ; s\right),
$$

where $d \Sigma_{\mu \nu}=\frac{1}{2} \frac{1}{(D-2) !} \epsilon_{\mu \nu \mu_{1} \mu_{2} \cdots \mu_{(D-2)}} d x^{\mu_{1}} \wedge \cdots \wedge d x^{\mu_{(D-2)}}$ with $\epsilon_{012 \cdots(D-1)}=\sqrt{-g}$ and $G_{(D)}$ is the gravitational constant in $D$ dimensions. Equation (2.24) can be regarded as a 
proposal of the formalism for the conserved charge, defined in the interior region or at the asymptotical infinity, for any covariant gravity theory with the Lagrangian (2.1) whenever its integration is well defined [30]. In contrast to another formula of conserved charges for covariant gravity theories in [32, 33, 34, which is defined in terms of the so-called solution phase space method building on basis of the LIW and BBC approaches, the formula (2.24) is equivalent with that one.

Finally, in contrast with the original ADT formulation [1, 2, 3], the formula (2.24) for the conserved charges is dependent on the gravitational and matter fields, as well as their fluctuations, while the usual ADT charges merely involve the gravitational field and its perturbation. Such a manner of the original ADT formulation may succeed to produce physical conserved charges when the matter fields fall off quite fast at infinity and they do not change the default asymptotic structure, which is the usual asymptotically (A)dS space. Otherwise, a serious consideration on the contributions from the matter fields becomes necessary to make the ADT formalism more universal, as in the present work. For example, the original ADT formulation fails to yield the physically meaningful mass of the black holes in Horndeski theory [17] and the Gödel-type black holes in five-dimensional minimal supergravity [41. However, the formula (2.24) is applicable since it contains the contributions from the matter fields. What is more, if the matter fields are taken into account, the ADT formalism naturally possesses the property that it is invariant under conformal transformation [14], but the usual ADT formalism requires the asymptotic condition that the conformal factor goes to unity at infinity to have the same property [44].

\section{Conserved charges of gravity theories with scalar fields and $p$-form potentials}

In this section, our main goal is to extend the general derivations in the previous section to systematically investigate the conserved charges of $D$-dimensional covariant gravity theories consisting of a gravitational field $g_{\mu \nu}, m$ scalar fields $\phi^{(k)}(k=1,2, \cdots, m)$ and a number

of $p$-form potentials $A_{(p)}$, where $1 \leq p \leq n-1$ and $2 \leq n \leq D$. In [31, 36, 37], the conserved charges of the gravity theories with $p$-form potentials were also investigated in other methods. The Lagrangian describing these theories is assumed to take the generic 
form

$$
\begin{aligned}
\mathcal{L} & =\mathcal{L}_{R}+\mathcal{L}_{\phi}+\mathcal{L}_{M} \\
\mathcal{L}_{R} & =\sqrt{-g} R \\
\mathcal{L}_{\phi} & =\sqrt{-g} L_{\phi}=\sqrt{-g}\left[\sum_{i, j=1}^{m} X_{i j}\left(\phi^{(k)}\right) \nabla^{\mu} \phi^{(i)} \nabla_{\mu} \phi^{(j)}+V\left(\phi^{(k)}\right)\right], \\
\mathcal{L}_{M} & =\sqrt{-g} L_{M}\left(g, \phi^{(k)}, A_{(p)}, F_{(p+1)}\right),
\end{aligned}
$$

where the $(p+1)$-form field strengths are defined by $F_{(p+1)}=d A_{(p)}$ and the pair of the indices $(i, j)$ in the function $X_{i j}$ are symmetric. In this work, without loss of generality, the function $L_{M}$ is supposed to possess two types of structures. The first one is

$$
\begin{aligned}
L_{M} \sim & W\left(\phi^{(k)}\right) g \cdots g F_{\left(q_{1}\right)} \cdots F_{\left(q_{J}\right)} A_{\left(p_{1}\right)} \cdots A_{\left(p_{I}\right)}, \\
& \left(p_{1}, \cdots, p_{I}=1, \cdots, n-1 ; q_{1}, \cdots, q_{J}=2, \cdots, n\right),
\end{aligned}
$$

and the second one is

$$
\begin{aligned}
L_{M}= & \sqrt{-g} W\left(\phi^{(k)}\right) g^{\mu_{1} \nu_{1}} \cdots g^{\mu_{D} \nu_{D}} \bar{\epsilon}_{\mu_{1} \cdots \mu_{D}} \times \\
& \left(F_{\left(q_{1}\right)} \cdots F_{\left(q_{t}\right)} A_{\left(p_{1}\right)} \cdots A_{\left(p_{s}\right)}\right)_{\nu_{1} \cdots \nu_{D}} .
\end{aligned}
$$

In the above equation, $\bar{\epsilon}_{\mu_{1} \cdots \mu_{D}}$ is the totally antisymmetric Levi-Civita tensor density with $\bar{\epsilon}_{01 \cdots(D-1)}=1$. The indices $p_{1}, \cdots, p_{s}=1, \cdots,(n-1)$ and the indices $q_{1}, \cdots, q_{t}=2, \cdots, n$. Equation (3.3) in general describes the conventional Chern-Simons-like terms involved in various gauge theories. In fact, the first structure of $L_{M}$ in Eq. (3.2) can be generally rewritten as a more concrete form, which is as follows:

$$
L_{M}=\mathbb{L}_{M}=W\left(\phi^{(k)}\right) H_{(N)}^{\mu_{1} \cdots \mu_{N}} Y_{(N) \mu_{1} \cdots \mu_{N}}, \quad 1 \leq N \leq D,
$$

where the two totally antisymmetric $N$-rank tensors $H_{(N)}$ and $Y_{(N)}$ are defined by

$$
\begin{aligned}
H_{(N) \mu_{1} \cdots \mu_{N}} & =\left(F_{\left(q_{1}\right)} \cdots F_{\left(q_{t}\right)} A_{\left(p_{1}\right)} \cdots A_{\left(p_{s}\right)}\right)_{\left[\mu_{1} \cdots \mu_{N}\right]}, \\
Y_{(N) \mu_{1} \cdots \mu_{N}} & =\left(F_{\left(\tilde{q}_{1}\right)} \cdots F_{\left(\tilde{q}_{j}\right)} A_{\left(\tilde{p}_{1}\right)} \cdots A_{\left(\tilde{p}_{i}\right)}\right)_{\left[\mu_{1} \cdots \mu_{N}\right]} .
\end{aligned}
$$

In the above equation, the integers $\tilde{p}_{1}, \cdots, \tilde{p}_{i}$ range from 1 to $(n-1)$ while the integers $\tilde{q}_{1}, \cdots, \tilde{q}_{j}$ range from 2 to $n$. In addition, all the indices $\left(p_{1}, \cdots, p_{s}, \tilde{p}_{1}, \cdots, \tilde{p}_{i}\right)$ and $\left(q_{1}, \cdots, q_{t}, \tilde{q}_{1}, \cdots, \tilde{q}_{j}\right)$ have to satisfy the following constraints

$$
\begin{aligned}
& N=\left(p_{1}+\cdots+p_{s}\right)+\left(q_{1}+\cdots+q_{t}\right), \\
& N=\left(\tilde{p}_{1}+\cdots+\tilde{p}_{i}\right)+\left(\tilde{q}_{1}+\cdots+\tilde{q}_{j}\right) .
\end{aligned}
$$


Among all the potentials in $H_{(N)}$ and $Y_{(N)}$, some or all of them are allowed to be equal to each other, as well as all the field strengths. However, in our analysis, all the potentials and field strengths are treated as being formally independent of each other. Furthermore, it is worth noting that the second structure in Eq. (3.3) can also be reexpressed as the form like the one in Eq. (3.4) if the Levi-Civita tensor density $\bar{\epsilon}_{\mu_{1} \cdots \mu_{D}}$ is understood as a $D$-form "potential", but an additional factor $\sqrt{-g}$ appears. In such a case, one will see that the procedure to derive the expected results, including the expressions of the equations of motion, currents and potentials, is parallel with the one for $L_{M}$ with the form (3.2) or (3.4) from appendix $\mathrm{A}$. To this point, the form (3.4) for $L_{M}$ is more general.

Let us pause to make a comment on the Lagrangian $\mathcal{L}_{M}$. Due to the forms of $L_{M}$ given in Eqs. (3.2), (3.3) and (3.4), one sees that the Lagrangian $\mathcal{L}_{M}$ is very general. It incorporates a broad class of terms consisting of scalar fields and $p$-form gauge fields for the Lagrangians in the context of a wide range of gravity theories with these matter fields, such as the Einstein-Maxwell-dilaton theories, the low-energy effective field theories of heterotic string theories, supergravity theories and so on. Particularly in the context of supergravity theories, the parts involving scalar fields and $p$-form potentials of the Lagrangian, describing the five-dimensional Einstein-Maxwell-Chern-Simons theory, the bosonic sector of the tendimensional type IIB supergravity, the bosonic part of the eleven-dimensional supergravity etc can be regarded as special cases of $\mathcal{L}_{M}$.

Varying the Lagrangian (3.1) with respect to the gravitational field, the scalar fields and the $p$-form potentials, one obtains

$$
\begin{aligned}
\delta \mathcal{L}= & \sqrt{-g}\left[\mathcal{E}_{\mu \nu}^{(g r)} \delta g^{\mu \nu}+\sum_{k=1}^{m} \breve{\mathcal{E}}_{(k)}^{(\phi)} \delta \phi^{(k)}+\sum_{p=1}^{n-1} \mathcal{E}_{(p)}^{(A) \mu_{1} \cdots \mu_{p}} \delta A_{(p) \mu_{1} \cdots \mu_{p}}\right. \\
& \left.+\nabla_{\mu} \Theta^{\mu}\left(\delta g, \delta \phi^{(k)}, \delta A_{(p)}\right)\right]
\end{aligned}
$$

where the expressions $\mathcal{E}_{(p)}^{(A) \mu_{1} \cdots \mu_{p}}$ of the field equations with respect to the gauge fields $A_{(p)}$ are given in Eq. (A.2), and the other ones for the gravitational field and scalar fields, as well as the surface term, are defined through

$$
\begin{aligned}
\mathcal{E}_{\mu \nu}^{(g r)} & =\mathcal{E}_{(R) \mu \nu}^{(g r)}+\mathcal{E}_{(\phi) \mu \nu}^{(g r)}+\mathcal{E}_{(M) \mu \nu}^{(g r)}, \\
\breve{\mathcal{E}}_{(k)}^{(\phi)} & =\tilde{\mathcal{E}}_{(k)}^{(\phi)}+\mathcal{E}_{(k)}^{(\phi)}, \\
\Theta^{\mu} & =\Theta_{(R)}^{\mu}+\Theta_{(\phi)}^{\mu}+\Theta_{(M)}^{\mu} .
\end{aligned}
$$


In the above equations, for the expressions $\mathcal{E}_{(M) \mu \nu}^{(g r)}$ and $\mathcal{E}_{(k)}^{(\phi)}$, as well as the boundary term $\Theta_{(M)}^{\mu}$, see Eq. (A.2). Both the $\mathcal{E}_{(\phi) \mu \nu}^{(g r)}$ and $\tilde{\mathcal{E}}_{(k)}^{(\phi)}$ expressions of the field equations and the surface term $\Theta_{(\phi)}^{\mu}$ are presented by Eqs. (A.19) and (A.20) respectively. In addition, the expression for the equation of motion $\mathcal{E}_{(R) \mu \nu}^{(g r)}$ and the boundary term $\Theta_{(R)}^{\mu}$, associated with the Lagrangian $\mathcal{L}_{R}$, are read off as

$$
\begin{aligned}
\mathcal{E}_{(R) \mu \nu}^{(g r)} & =G_{\mu \nu}=R_{\mu \nu}-\frac{1}{2} g_{\mu \nu} R, \\
\Theta_{(R)}^{\mu} & =2 g^{\mu[\rho} g^{\nu] \sigma} \nabla_{\nu} \delta g_{\rho \sigma}=\nabla^{\mu}\left(g_{\rho \sigma} \delta g^{\rho \sigma}\right)-\nabla_{\rho} \delta g^{\rho \mu} .
\end{aligned}
$$

According to Eq. (2.6), the off-shell Noether current with respect to the Lagrangian (3.1) is defined by

$$
\begin{aligned}
J^{\mu} & =2 \mathcal{E}^{\mu \nu} \zeta_{\nu}+\zeta^{\mu} L-\Theta^{\mu}\left(\mathcal{L}_{\zeta} g, \mathcal{L}_{\zeta} \phi^{(k)}, \mathcal{L}_{\zeta} A_{(p)}\right) \\
& =J_{(R)}^{\mu}+J_{(\phi)}^{\mu}+J_{(M)}^{\mu} .
\end{aligned}
$$

In Eq. (3.12), the $J_{(R)}^{\mu}$ current, corresponding to the Lagrangian $\mathcal{L}_{R}$, is defined through

$$
J_{(R)}^{\mu}=2 \mathcal{E}_{(R)}^{(g r) \mu \nu} \zeta_{\nu}+\zeta^{\mu} L_{R}-\Theta_{(R)}^{\mu}\left(\mathcal{L}_{\zeta} g\right)
$$

Its corresponding potential $K_{(R)}^{\mu \nu}$ has the form

$$
K_{(R)}^{\mu \nu}=2 \nabla^{[\mu} \zeta^{\nu]}
$$

the $J_{(\phi)}^{\mu}$ current given by Eq. (A.23) takes a zero value, that is, $J_{(\phi)}^{\mu}=0$, and the $J_{(M)}^{\mu}$ current, associated with the Lagrangian $\mathcal{L}_{M}$, is presented by (A.12). The quantity $\mathcal{E}^{\mu \nu}$ is given by

$$
\mathcal{E}^{\mu \nu}=\mathcal{E}_{(R)}^{(g r) \mu \nu}+\mathcal{E}_{(\phi)}^{(g r) \mu \nu}+\mathcal{E}_{(M)}^{\mu \nu},
$$

where the specific expression for $\mathcal{E}_{(M)}^{\mu \nu}$ can be found in Eq. (A.11). The Noether current $J^{\mu}$ in Eq. (3.12) further yields the off-shell Noether potential

$$
\begin{aligned}
K_{R \phi M}^{\mu \nu} & =K_{(R)}^{\mu \nu}+K_{(\phi)}^{\mu \nu}+K_{(M)}^{\mu \nu} \\
& =2 \nabla^{[\mu} \zeta^{\nu]}-\sum_{p=1}^{n-1} p(p+1) U_{(p+1)}^{\mu \nu \mu_{1} \cdots \mu_{(p-1)}} \zeta^{\sigma} A_{(p) \sigma \mu_{1} \cdots \mu_{(p-1)}},
\end{aligned}
$$

where $K_{(\phi)}^{\mu \nu}=0$ and the potential $K_{(M)}^{\mu \nu}$, associated with the Lagrangian $\mathcal{L}_{M}$, is determined by Eq. (A.13). 
Next, like before, supposing that the spacetime admits a Killing vector $\xi^{\mu}$ that obeys all the conditions demanded in Eq. (2.8), the off-shell ADT potential $Q_{R \phi M}^{\mu \nu}$ involving this Killing vector is given in terms of the Noether potential $K_{R \phi M}^{\mu \nu}$ in Eq. (3.16) and the $\Theta^{\mu}\left(\delta g, \delta \phi^{(k)}, \delta A_{(p)}\right)$ surface term in Eq. (3.10) by

$$
\begin{aligned}
Q_{R \phi M}^{\mu \nu} & =\frac{1}{2} \frac{1}{\sqrt{-g}} \delta\left(\sqrt{-g} K_{R \phi M}^{\mu \nu}(\xi)\right)-\xi^{[\mu} \Theta^{\nu]}\left(\delta g, \delta \phi^{(k)}, \delta A_{(p)}\right) \\
& =Q_{(R)}^{\mu \nu}+Q_{(\phi)}^{\mu \nu}+Q_{(M)}^{\mu \nu}
\end{aligned}
$$

where the total potential $Q_{R \phi M}^{\mu \nu}$ is accordingly split into three parts, i.e., the potentials $Q_{(R)}^{\mu \nu}, Q_{(\phi)}^{\mu \nu}$ and $Q_{(M)}^{\mu \nu}$, corresponding to the contributions from the Lagrangian $\mathcal{L}_{R}, \mathcal{L}_{\phi}$ and $\mathcal{L}_{M}$ respectively, are given by

$$
\begin{aligned}
Q_{(R)}^{\mu \nu} & =\frac{1}{2} \frac{1}{\sqrt{-g}} \delta\left(\sqrt{-g} K_{(R)}^{\mu \nu}(\xi)\right)-\xi^{[\mu} \Theta_{(R)}^{\nu]}(\delta g), \\
Q_{(\phi)}^{\mu \nu} & =-\xi^{[\mu} \Theta_{(\phi)}^{\nu]}\left(\delta \phi^{(k)}\right), \\
Q_{(M)}^{\mu \nu} & =\frac{1}{2} \frac{1}{\sqrt{-g}} \delta\left(\sqrt{-g} K_{(M)}^{\mu \nu}(\xi)\right)-\xi^{[\mu} \Theta_{(M)}^{\nu]}\left(\delta A_{(p)}\right) .
\end{aligned}
$$

The off-shell ADT current $\mathcal{J}_{R \phi M}^{\mu}$ for the Lagrangian (3.1) can be got through the relation $\mathcal{J}_{R \phi M}^{\mu}=\nabla_{\nu} Q_{R \phi M}^{\mu \nu}$. When $\mathbb{L}_{M}$ in Eq. (3.4) is adopted to take the place of the quantity $L_{M}$ of the Lagrangian $\mathcal{L}_{M}$ in the total Lagrangian (3.1), the ADT potential $Q_{(M)}^{\mu \nu}$ can be substituted by $Q_{\left(\mathbb{L}_{M}\right)}^{\mu \nu}$, which is defined by

$$
Q_{\left(\mathbb{L}_{M}\right)}^{\mu \nu}=\frac{1}{2} \frac{1}{\sqrt{-g}} \delta\left(\sqrt{-g} K_{\left(\mathbb{L}_{M}\right)}^{\mu \nu}(\xi)\right)-\xi^{[\mu} \Theta_{\left(\mathbb{L}_{M}\right)}^{\nu]}\left(\delta A_{(p)}\right)
$$

where the surface term $\Theta_{\left(\mathbb{L}_{M}\right)}^{\mu}$ and the off-shell Noether potential $K_{\left(\mathbb{L}_{M}\right)}^{\mu \nu}$ can be found in Eqs. (A.14) and (A.17) respectively. Furthermore, substituting the off-shell ADT potential (3.17) into Eq. (2.24), one obtains the formula for the conserved charge associated with the Lagrangian (3.1).

As we have mentioned in the previous section, the off-shell ADT potential (3.17) is essentially equivalent with those derived through the LIW method [24, 25, 26] and the BBC method [27, 28, 29, 30] respectively. For example, when Eq. (3.17) is applied to compute the off-shell ADT potential for the gravity theory with a single $p$-form potential given in the works [36] and [37], where potentials for this theory were derived through the LIW approach and the BBC method respectively, one finds that all the potentials agree with each other. On the other hand, as a consequence of the generality for the Lagrangian $\mathcal{L}_{M}$, the 
potential (3.17) can be widely used to compute the conserved charges of gravity theories in the presence of scalar fields and $p$-form gauge fields. Its typical applications are to calculate the conserved charges of black holes in Einstein-Maxwell-dilaton theory and supergravity theories [38, 39, 40, 41, 42, 43, such as the dyonic AdS black holes in four-dimensional maximal $\mathcal{N}=8, S O(8)$ gauged supergravity [38], the general nonextremal rotating charged AdS black holes in five-dimensional $U(1)^{3}$ gauged supergravity [39], the rotating charged Kaluza-Klein black holes [40] and the rotating charged Gödel-type black holes [41] in fivedimensional minimal supergravity, the general nonextremal charged rotating black holes in five-dimensional minimal gauged supergravity [42], and so on. If the formula (2.24), endowed with the off-shell ADT potential (3.17), is applied to compute the conserved charges within the low-energy effective field theory describing heterotic string theory like in [14], one can observe that all the results in that work are covered.

\section{Conserved charges of $(\mathrm{D}+1)$-dimensional Einstein gravity under Kaluza-Klein reduction}

In this section, we investigate the behaviour of conserved charges in $(D+1)$-dimensional general relativity, which is defined through the off-shell generalized ADT potential, by performing a Kaluza-Klein reduction procedure along a compactified direction with $S^{1}$ topology. As is known to us, the $(D+1)$-dimensional Einstein gravity is described by the Einstein-Hilbert Lagrangian

$$
\hat{\mathcal{L}}_{\hat{R}}=\sqrt{-\hat{g}} \hat{R}
$$

Here and in what follows, all the quantities with the hat " " " correspond to the ones in $(D+1)$-dimensional spacetime. It is assumed that the $(D+1)$-dimensional spacetime manifold $M^{D+1}$ is the direct product of a $D$-dimensional spacetime manifold $M^{D}$ and an $S^{1}$ circle, namely, $M^{D+1}=M^{D} \otimes S^{1}$. The $(D+1)$-dimensional spacetime may be endowed with the coordinate system $\hat{x}^{\hat{\mu}}=\left(x^{\mu}, z\right)$, where $x^{\mu}$, parametrizing the manifold $M^{D}$, runs over all the coordinates in $D$ dimensions and the $z$ coordinate indicates the compactified direction on the $S^{1}$ circle of radius $L$. By further introducing some $D$-dimensional fields, which are the metric tensor $g_{\mu \nu}$, the Kaluza-Klein vector $\mathcal{A}_{\mu}$ and the dilaton $\varphi$ respectively, 
the $(D+1)$-dimensional metric ansatz is supposed to take the following form [35]:

$$
\begin{aligned}
d \hat{s}_{(D+1)}^{2} & =e^{2 \alpha \varphi} d s_{(D)}^{2}+e^{2 \beta \varphi}(d z+\mathcal{A})^{2}, \\
d s_{(D)}^{2} & =g_{\mu \nu} d x^{\mu} d x^{\nu},
\end{aligned}
$$

where the 1 -form gauge field reads $\mathcal{A}=\mathcal{A}_{\mu} d x^{\mu}$, while both the two dimensionally dependent constants $\alpha$ and $\beta$ are given by

$$
\beta=-(D-2) \alpha, \quad \alpha^{2}=\frac{1}{2(D-1)(D-2)} .
$$

It is supposed that all the components of the $(D+1)$-dimensional metric tensor $\hat{g}_{\hat{\mu} \hat{\nu}}$ do not include the $z$ coordinate as a variable. As a result, all the $D$-dimensional fields, such as $g_{\mu \nu}, \mathcal{A}_{\mu}$ and $\varphi$, are independent of the coordinate $z$. By performing a Kaluza-Klein dimensional reduction of the $(D+1)$-dimensional ansatz (4.2) along the $z$ direction, the $(D+1)$-dimensional Einstein-Hilbert Lagrangian (4.1), which only consists of the $(D+1)$ dimensional metric tensor $\hat{g}_{\hat{\mu} \hat{\nu}}$, can be reexpressed in terms of all the $D$-dimensional fields as

$$
\begin{aligned}
\hat{\mathcal{L}}_{\hat{R}} & =\mathcal{L}_{E M D}=\sqrt{-g} L_{E M D} \\
& =\sqrt{-g}\left(R-\frac{1}{2} \nabla^{\mu} \varphi \nabla_{\mu} \varphi-\frac{1}{4} e^{-2(D-1) \alpha \varphi} \mathcal{F}^{2}\right),
\end{aligned}
$$

where the 2 -form field strength $\mathcal{F}=d \mathcal{A}$. Note that we have dropped the $\square \varphi$ term that makes no contribution to the field equations and one can strictly prove that this term also contributes nothing to the off-shell ADT potential. The lower-dimensional Lagrangian $\mathcal{L}_{E M D}$ is the one describing the Einstein-Maxwell-dilaton theory for a particular value $-2(D-1) \alpha$ of dilaton coupling. Equation (4.4) demonstrates that the $(D+1)$-dimensional Einstein gravity is essentially equivalent to the $D$-dimensional theory equipped with a gravitational field, a scalar and a $U(1)$ gauge field.

Letting $\hat{\xi}^{\hat{\mu}}$ denote a $(D+1)$-dimensional Killing vector, according to Eq. (3.18), an off-shell ADT potential for the Einstein-Hilbert Lagrangian $\hat{\mathcal{L}}_{\hat{R}}$ takes the form

$$
\hat{Q}_{(\hat{R})}^{\hat{\mu} \hat{\nu}}=\frac{1}{2} \frac{1}{\sqrt{-\hat{g}}} \delta\left(\sqrt{-\hat{g}} \hat{K}_{(\hat{R})}^{\hat{\mu} \hat{\nu}}(\hat{\xi})\right)-\hat{\xi}^{[\hat{\mu}} \hat{\Theta}_{(\hat{R})}^{\hat{\nu}]}(\delta \hat{g}) .
$$

On the other hand, with help of Eq. (3.17), the ADT potential related to the $D$-dimensional Lagrangian $\mathcal{L}_{E M D}$ is given by

$$
Q_{E M D}^{\mu \nu}=\frac{1}{2} \frac{1}{\sqrt{-g}} \delta\left(\sqrt{-g} K_{E M D}^{\mu \nu}(\xi)\right)-\xi^{[\mu} \Theta_{E M D}^{\nu]}(\delta g, \delta \varphi, \delta \mathcal{A})
$$


where the $K_{E M D}^{\mu \nu}$ potential, which can be deduced from Eq. (3.16), and the surface term $\Theta_{E M D}^{\mu}$ are presented by

$$
\begin{aligned}
K_{E M D}^{\mu \nu}= & 2 \nabla^{[\mu} \xi^{\nu]}+e^{-2(D-1) \alpha \varphi} \xi^{\sigma} \mathcal{A}_{\sigma} \mathcal{F}^{\mu \nu} \\
\Theta_{E M D}^{\mu}= & \nabla^{\mu}\left(g_{\rho \sigma} \delta g^{\rho \sigma}\right)-\nabla_{\rho} \delta g^{\rho \mu} \\
& -\left(\nabla^{\mu} \varphi\right) \delta \varphi-e^{-2(D-1) \alpha \varphi} \mathcal{F}^{\mu \nu} \delta \mathcal{A}_{\nu}
\end{aligned}
$$

respectively. The vector $\xi^{\mu}$ in Eq. (4.7) is the $D$-dimensional Killing vector, reflecting the symmetry of spacetime and satisfying

$$
\mathcal{L}_{\xi} g_{\mu \nu}=0, \quad \mathcal{L}_{\xi} \varphi=0, \quad \mathcal{L}_{\xi} \mathcal{A}=0
$$

In the Appendix $\mathbf{C}$, we have proved that the $\hat{\xi}_{(b)}^{\hat{\mu}}$ vector, defined through $\hat{\xi}_{(b)}^{\hat{\mu}}=\left(\xi^{\mu}, b\right)$, is a Killing vector in $(D+1)$ dimensions, where the parameter $b$ is an arbitrary constant, if the $D$-dimensional $\xi^{\mu}$ vector satisfies Eq. (4.8). The $\hat{\xi}_{(b)}^{\hat{\mu}}$ vector is associated with the off-shell Noether potential $\hat{K}_{(\hat{R})}^{\hat{\mu} \hat{\nu}}\left(\hat{\xi}_{(b)}\right)=\hat{K}_{(\hat{R})}^{\hat{\mu} \hat{\nu}}\left(\hat{\xi} \rightarrow \hat{\xi}_{(b)}\right)$, whose $(\mu, \nu)$-component is reexpressed in terms of all the lower-dimensional fields as

$$
\hat{K}_{(\hat{R})}^{\mu \nu}\left(\hat{\xi}_{(b)}\right)=e^{-2 \alpha \varphi} K_{E M D}^{\mu \nu}+2 \xi^{[\mu} \nabla^{\nu]} e^{-2 \alpha \varphi}+b e^{-2 D \alpha \varphi} \mathcal{F}^{\mu \nu}
$$

To derive Eq. (4.9), we have made use of Eq. (C.3). On the other hand, by utilizing Eq. (B.8), one finds that the $\mu$-component of the $(D+1)$-dimensional surface term $\hat{\Theta}_{(\hat{R})}^{\hat{\mu}}$ and the $D$-dimensional one $\Theta_{E M D}^{\mu}$ has the following relationship

$$
\hat{\Theta}_{(\hat{R})}^{\mu}=e^{-2 \alpha \varphi} \Theta_{E M D}^{\mu}+\frac{e^{-2 \alpha \varphi}}{\sqrt{-g}} \delta\left(\sqrt{-g} e^{2 \alpha \varphi} \nabla^{\mu} e^{-2 \alpha \varphi}\right) .
$$

Hence, when $\hat{\xi}_{(b)}^{\hat{\mu}}=\hat{\xi}_{(0)}^{\hat{\mu}}=\left(\xi^{\mu}, 0\right)$, Eqs. (4.9) and (4.10) yield

$$
\sqrt{-\hat{g}} \hat{Q}_{(\hat{R})}^{\mu \nu}\left(\hat{\xi}_{(0)}\right)=\sqrt{-g} Q_{E M D}^{\mu \nu}+\sqrt{-g} e^{2 \alpha \varphi} \delta \xi^{[\mu} \nabla^{\nu]} e^{-2 \alpha \varphi} .
$$

In Eq. (4.11), the $(\mu, \nu)$-component of the $(D+1)$-dimensional off-shell ADT potential multiplied by the factor $\sqrt{-\hat{g}}$ coincides with the $D$-dimensional ADT potential multiplied by the factor $\sqrt{-g}$, if the variation of the Killing vector vanishes, that is, $\delta \xi^{\mu}=0$. This is always guaranteed when the Killing vectors are associated with the mass and angular momentum. On the other hand, when $\hat{\xi}_{(b)}^{\hat{\mu}}=\hat{\xi}_{(1)}^{\hat{\mu}}=(0, \cdots, 0,1)$, one gets

$$
\sqrt{-\hat{g}} \hat{Q}_{(\hat{R})}^{\mu \nu}\left(\hat{\xi}_{(1)}\right)=\frac{1}{2} \delta\left(\sqrt{-g} e^{-2(D-1) \alpha \varphi} \mathcal{F}^{\mu \nu}\right) .
$$


On basis of Eq. (4.11), together with help of the formula (2.24) for the conserved charge and the relationship between the $(D+1)$-dimensional gravitational constant $\hat{G}_{(D+1)}$ and the one $G_{(D)}$ in $D$ dimensions, namely,

$$
\hat{G}_{(D+1)}=2 \pi L G_{(D)},
$$

where $L$ is the radius of the $S^{1}$ circle, one observes that the conserved charges in $(D+1)$ dimensions coincide with the ones in $D$ dimensions. That is to say, the conserved charge defined in terms of the off-shell generalized ADT potential is invariant under Kaluza-Klein dimensional reduction in the framework of the theory for Einstein gravity. It has been mentioned that the off-shell generalized ADT method is equivalent with the LIW and BBC methods, so the conserved charges defined via these methods also exhibit the property that they are invariant under Kaluza-Klein reduction along a compactified direction. On the other hand, according to the formula (2.24), the conserved charge associated with the Killing vector $\hat{\xi}_{(1)}^{\hat{\mu}}$ is defined by

$$
\mathcal{Q}_{z}=\frac{1}{32 \pi(D-2) ! G_{(D)}} \int_{\partial \Sigma} e^{-2(D-1) \alpha \varphi} \mathcal{F}^{\mu \nu} \epsilon_{\mu \nu \mu_{1} \mu_{2} \cdots \mu_{(D-2)}} d x^{\mu_{1}} \wedge \cdots \wedge d x^{\mu_{(D-2)}},
$$

where $\partial \Sigma$ is the boundary of a $(D-1)$-dimensional hypersurface $\Sigma$. The charge $\mathcal{Q}_{z}$ is nothing but the electric charge with respect to the Kaluza-Klein vector $\mathcal{A}_{\mu}$ in the $D$-dimensional Einstein-Maxwell-dilaton theory described by the Lagrangian (4.4). As a consequence, we conclude that the angular momentum along the $z$ direction in the context of the higherdimensional Einstein gravity theory is just the electric charge associated with the KaluzaKlein vector in the lower-dimensional theory when the conserved charge is defined in terms the off-shell ADT formalism.

\section{Properties for conserved charges in gravity theories with n-form field strength under Kaluza-Klein reduction}

In this section, we extend the analysis in the previous section to investigate the properties of conserved charges for gravity theories including a gravitational filed, a single $n$-form field strength and $m$ scalar field $\phi^{(k)}(k=1, \cdots, m)$ by performing Kaluza-Klein dimensional reduction along a compactified direction on a circle. In comparison with the case of Einstein gravity, we wonder whether the conserved charge will be influenced by the Kaluza-Klein reduction when the matter fields are included. 
Without loss of generality, we take into consideration the $(D+1)$-dimensional Lagrangian that has the form

$$
\begin{aligned}
\hat{\mathcal{L}}_{\hat{R} \hat{F}} & =\hat{\mathcal{L}}_{\hat{R}}+\hat{\mathcal{L}}_{\hat{F}}+\hat{\mathcal{L}}_{\phi}, \\
\hat{\mathcal{L}}_{\hat{F}} & =\sqrt{-\hat{g}} \gamma\left(\phi^{(k)}\right) \hat{F}_{(n)}^{2}, \\
\hat{\mathcal{L}}_{\phi} & =\sqrt{-\hat{g}}\left[\sum_{i, j=1}^{m} X_{i j}\left(\phi^{(k)}\right) \hat{\nabla}^{\hat{\mu}} \phi^{(i)} \hat{\nabla}_{\hat{\mu}} \phi^{(j)}+V\left(\phi^{(k)}\right)\right],
\end{aligned}
$$

where the Lagrangian $\hat{\mathcal{L}}_{\hat{R}}$ is given in Eq. (4.1), and the $n$-form field strength $\hat{F}_{(n)}=d \hat{A}_{(n-1)}$. What is more, in Eq. (5.1), the $(n-1)$-form potential $\hat{A}_{(n-1)}$ in $(D+1)$ dimensions is defined in terms of the $D$-dimensional gauge fields $A_{(n-1)}$ and $A_{(n-2)}$ by

$$
\begin{aligned}
& \hat{A}_{(n-1) \mu_{1} \cdots \mu_{(n-1)}}=A_{(n-1) \mu_{1} \cdots \mu_{(n-1)}}, \\
& \hat{A}_{(n-1) \mu_{1} \cdots \mu_{(n-2)} z}=A_{(n-2) \mu_{1} \cdots \mu_{(n-2)}} .
\end{aligned}
$$

From Eq. (5.2), one easily gets all the components of the $\hat{F}_{(n)}$ field strength that are

$$
\begin{aligned}
\hat{F}_{(n) \mu_{1} \cdots \mu_{n}} & =F_{(n) \mu_{1} \cdots \mu_{n}} \\
& =n \nabla_{\left[\mu_{1}\right.} A_{\left.(n-1) \mu_{2} \cdots \mu_{n}\right]}, \\
\hat{F}_{(n) \mu_{1} \cdots \mu_{(n-1)} z} & =F_{(n-1) \mu_{1} \cdots \mu_{(n-1)}} \\
& =(n-1) \nabla_{\left[\mu_{1}\right.} A_{\left.(n-2) \mu_{2} \cdots \mu_{(n-1)}\right]} .
\end{aligned}
$$

In the present case, the $(D+1)$-dimensional metric ansatz is assumed to take the same form as the one in Eq. (4.2). Besides, all the fields, including the metric tensor $\hat{g}_{\hat{\mu} \hat{\nu}}$, the $A_{(n-1)}$ and $A_{(n-2)}$ potentials, and the scalar fields $\phi^{(k)}$, are assumed to have no dependence on the $z$ coordinate. Obviously, both the lower-dimensional $F_{(n)}$ and $F_{(n-1)}$ strengths, together with the functions $X_{i j}, V$ and $\gamma$, are independent of the $z$ coordinate as well.

Now, following the general derivation for the Lagrangian with multiple scalar fields and $p$-form potentials in Appendix $\mathbf{D}$ to perform Kaluza-Klein dimensional reduction along the $z$ direction on basis of the $(D+1)$-dimensional ansatz (4.2), we further send the Lagrangian (5.1) to the form

$$
\begin{aligned}
\hat{\mathcal{L}}_{\hat{R} \hat{F}} & =\mathcal{L}_{R F} \\
& =\mathcal{L}_{E M D}+\mathcal{L}_{F}+\tilde{\mathcal{L}}_{\phi},
\end{aligned}
$$


where the quantities $\mathcal{L}_{F}$ and $\tilde{\mathcal{L}}_{\phi}$ are given by

$$
\begin{aligned}
& \mathcal{L}_{F}=\sqrt{-g} \gamma\left(e^{-2(n-1) \alpha \varphi} \tilde{F}_{(n)}^{2}+n e^{2(D-n) \alpha \varphi} F_{(n-1)}^{2}\right), \\
& \tilde{\mathcal{L}}_{\phi}=\sqrt{-g}\left(\sum_{i, j=1}^{m} X_{i j} \nabla^{\mu} \phi^{(i)} \nabla_{\mu} \phi^{(j)}+e^{2 \alpha \varphi} V\right)
\end{aligned}
$$

and see Eq. (4.4) for the expression of $\mathcal{L}_{E M D}$. In Eq. (5.5), the $n$-form field strength $\tilde{F}_{(n)}$ is defined by

$$
\tilde{F}_{(n) \mu_{1} \cdots \mu_{n}}=F_{(n) \mu_{1} \cdots \mu_{n}}-n F_{(n-1)\left[\mu_{1} \cdots \mu_{(n-1)}\right.} \mathcal{A}_{\left.\mu_{n}\right]} .
$$

Note that here $d \tilde{F}_{(n)} \neq 0$.

Next, we pay attention to calculating the off-shell ADT potentials for the Lagrangians $\mathcal{L}_{F}$ and $\hat{\mathcal{L}}_{\hat{F}}$. As before, we introduce a Killing vector $\xi^{\mu}$ in the $D$-dimensional spacetime, which also satisfies the following equations

$$
\begin{aligned}
\mathcal{L}_{\xi} A_{(n-2)} & =0, & & \mathcal{L}_{\xi} A_{(n-1)}=0, \\
\mathcal{L}_{\xi} \phi^{(k)} & =0, & & (k=1, \cdots, m),
\end{aligned}
$$

apart from the ones in (4.8). According to Eq. (3.20), the $\xi^{\mu}$ Killing vector is in one-to-one correspondence with the ADT potential for the $D$-dimensional Lagrangian $\mathcal{L}_{F}$, which takes the form

$$
Q_{(F)}^{\mu \nu}=\frac{1}{2} \frac{1}{\sqrt{-g}} \delta\left(\sqrt{-g} K_{(F)}^{\mu \nu}(\xi)\right)-\xi^{[\mu} \Theta_{(F)}^{\nu]}\left(\delta A_{(n-2)}, \delta A_{(n-1)}\right),
$$

in which the off-shell Noether potential $K_{(F)}^{\mu \nu}(\xi)$, defined through Eq. (A.13), are read off as

$$
\begin{aligned}
K_{(F)}^{\mu \nu}(\xi)= & -2 n(n-1) \gamma e^{-2(n-1) \alpha \varphi} \xi^{\sigma}\left\{\tilde{F}_{(n)}^{\mu \nu \mu_{1} \cdots \mu_{(n-2)}} \times\right. \\
& {\left[A_{\sigma \mu_{1} \cdots \mu_{(n-2)}}^{(n-1)}-(n-2) \mathcal{A}_{\mu_{(n-2)}} A_{\sigma \mu_{1} \cdots \mu_{(n-3)}}^{(n-2)}\right] } \\
& \left.+(n-2) e^{2(D-1) \alpha \varphi} F_{(n-1)}^{\mu \nu \mu_{1} \cdots \mu_{(n-3)}} A_{\sigma \mu_{1} \cdots \mu_{(n-3)}}^{(n-2)}\right\},
\end{aligned}
$$

and the surface term $\Theta_{(F)}^{\mu}$, defined by Eq. (A.2), is expressed as

$$
\begin{aligned}
\Theta_{(F)}^{\mu}= & 2 n \gamma e^{-2(n-1) \alpha \varphi}\left\{\tilde{F}_{(n)}^{\mu \mu_{1} \cdots \mu_{(n-1)}} \times\right. \\
& {\left[\delta A_{\mu_{1} \cdots \mu_{(n-1)}}^{(n-1)}-(n-1) \mathcal{A}_{\mu_{(n-1)}} \delta A_{\mu_{1} \cdots \mu_{(n-2)}}^{(n-2)}\right] } \\
& \left.+(n-1) e^{2(D-1) \alpha \varphi} F_{(n-1)}^{\mu \mu_{1} \cdots \mu_{(n-2)}} \delta A_{\mu_{1} \cdots \mu_{(n-2)}}^{(n-2)}\right\}
\end{aligned}
$$


In the parallel analysis, with help of Eq. (3.20), the off-shell ADT potential for the $(D+1)$ dimensional Lagrangian $\hat{\mathcal{L}}_{\hat{F}}$ is given by

$$
\hat{Q}_{(\hat{F})}^{\hat{\mu} \hat{\nu}}=\frac{1}{2} \frac{1}{\sqrt{-\hat{g}}} \delta\left(\sqrt{-\hat{g}} \hat{K}_{(\hat{F})}^{\hat{\mu} \hat{\nu}}(\hat{\xi})\right)-\hat{\xi}^{[\hat{\mu}} \hat{\Theta}_{(\hat{F})}^{\hat{\nu}]}\left(\delta \hat{A}_{(n-1)}\right),
$$

where

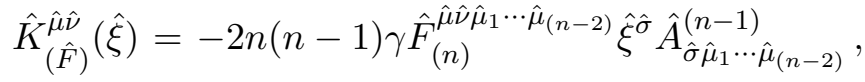

$$
\begin{aligned}
& \hat{\Theta}_{(\hat{F})}^{\hat{\mu}}=2 n \gamma \hat{F}_{(n)}^{\hat{\mu}^{\hat{\mu} \mu_{1} \cdots \hat{\mu}_{(n-1)}} \delta \hat{A}_{\hat{\mu}_{1} \cdots \hat{\mu}_{(n-1)}}^{(n-1)},},
\end{aligned}
$$

taking relatively simple forms.

As what has been done in the previous section, here we still expect to get the exact relationship between the ADT potentials for the Lagrangians $\mathcal{L}_{F}$ and $\hat{\mathcal{L}}_{\hat{F}}$. In order to do this, we first compare the surface terms yielded by the variation of both the Lagrangians. The $\mu$-component of the $(D+1)$-dimensional surface term $\hat{\Theta}_{(\hat{F})}^{\hat{\mu}}$ is presented in terms of all the lower-dimensional fields by

$$
\begin{aligned}
\hat{\Theta}_{(\hat{F})}^{\mu}= & 2 n \gamma \hat{g}^{\mu \nu} \hat{F}_{(n) \nu} \hat{\mu}_{1} \cdots \hat{\mu}_{(n-1)} \hat{A}_{\hat{\mu}_{1} \cdots \hat{\mu}_{(n-1)}}^{n-1)} \\
& +2 n \gamma \hat{g}^{\mu z} \hat{F}_{(n) z} \hat{\mu}_{1} \cdots \hat{\mu}_{(n-1)} \hat{A}_{\hat{\mu}_{1} \cdots \hat{\mu}_{(n-1)}(n-1)} \\
= & e^{-2 \alpha \varphi} \Theta_{(F)}^{\mu},
\end{aligned}
$$

in which Eq. (D.3) in the Appendix $\mathbf{D}$ has been used in order to get the last identity. On the other hand, the $(D+1)$-dimensional off-shell Nother potential $\hat{K}_{(\hat{F})}^{\mu \nu}\left(\hat{\xi}_{(b)}\right)$, where the $(D+1)$ dimensional Killing vector $\hat{\xi}_{(b)}^{\hat{\mu}}$ has the value $\left(\xi^{\mu}, b\right)$ and both the Eqs. (C.8) and (C.9) show that the Lie derivative of the $\hat{A}_{(n-1)}$ potential along this vector vanishes, is connected with the $D$-dimensional potential $K_{(F)}^{\mu \nu}(\xi)$ through Eq. (E.6). Hence, by combining Eq. (5.13) with Eq. (E.6), one finds that

$$
\sqrt{-\hat{g}} \hat{Q}_{(\hat{F})}^{\mu \nu}\left(\hat{\xi}_{(0)}\right)=\sqrt{-g} Q_{(F)}^{\mu \nu}
$$

when the Killing vector $\hat{\xi}_{(b)}^{\hat{\mu}}=\hat{\xi}_{(0)}^{\hat{\mu}}=\left(\xi^{\mu}, 0\right)$.

Making use of (3.19) , one observes that the $\operatorname{ADT}$ potentials $\hat{Q}_{(\phi)}^{\mu \nu}$ and $\tilde{Q}_{(\phi)}^{\mu \nu}$, associated with $\hat{\mathcal{L}}_{\phi}$ and $\tilde{\mathcal{L}}_{\phi}$ respectively, are related to each other through

$$
\begin{aligned}
\sqrt{-\hat{g}} \hat{Q}_{(\phi)}^{\mu \nu}\left(\hat{\xi}_{(0)}\right) & =\sqrt{-g} \tilde{Q}_{(\phi)}^{\mu \nu}\left(\hat{\xi}_{(0)}\right) \\
& =-\sqrt{-g} \xi^{[\mu} \Theta_{(\phi)}^{\nu]}\left(\delta \phi^{(k)}\right),
\end{aligned}
$$


in which the surface term $\Theta_{(\phi)}^{\mu}$ is presented in Eq. (A.20). On basis of Eqs. (4.11), (5.14) and (5.15), one further obtains the relationship between the total ADT potential $\hat{Q}_{(\hat{R} \hat{F})}^{\mu \nu}$ for the higher-dimensional Lagrangian $\mathcal{L}_{\hat{R} \hat{F}}$ and the one $Q_{(R F)}^{\mu \nu}$ for the lower-dimensional Lagrangian $\mathcal{L}_{R F}$, that is,

$$
\begin{aligned}
\sqrt{-\hat{g}} \hat{Q}_{(\hat{R} \hat{F})}^{\mu \nu}\left(\hat{\xi}_{(0)}\right) & =\sqrt{-\hat{g}}\left[\hat{Q}_{(\hat{R})}^{\mu \nu}\left(\hat{\xi}_{(0)}\right)+\hat{Q}_{(\hat{F})}^{\mu \nu}\left(\hat{\xi}_{(0)}\right)+\hat{Q}_{(\phi)}^{\mu \nu}\left(\hat{\xi}_{(0)}\right)\right] \\
& =\sqrt{-g}\left(Q_{E M D}^{\mu \nu}+Q_{(F)}^{\mu \nu}+\tilde{Q}_{(\phi)}^{\mu \nu}\right) \\
& =\sqrt{-g} Q_{(R F)}^{\mu \nu} .
\end{aligned}
$$

To arrive at the second equality in Eq. (5.16), the variation of the Killing vector $\xi^{\mu}$ is required to vanish since $\sqrt{-\hat{g}} \hat{Q}_{(\hat{R})}^{\mu \nu}=\sqrt{-g} Q_{E M D}^{\mu \nu}$ holds under the condition that $\delta \xi^{\mu}=0$. Generally speaking, the variation of the Killing vector with respect to mass or angular momentum always disappears. As the case of Einstein gravity in the previous section, from Eq. (5.16), we are able to see that the conserved charges defined through the formula (2.24) are unchanged before and after performing Kaluza-Klein reduction to the gravity theory described by the Lagrangian (5.1).

Besides, when the Killing vector $\hat{\xi}_{(b)}^{\hat{\mu}}=\hat{\xi}_{(1)}^{\hat{\mu}}=(0, \cdots, 0,1)$, with help of Eq. (E.6), the off-shell ADT potential associated with this vector is read off as

$$
\begin{aligned}
\sqrt{-\hat{g}} \hat{Q}_{(\hat{R} \hat{F})}^{\mu \nu}\left(\hat{\xi}_{(1)}\right)= & -n(n-1) \delta\left(\sqrt{-g} \gamma e^{-2(n-1) \alpha \varphi} \tilde{F}_{(n)}^{\mu \mu_{1} \cdots \mu_{(n-2)} \nu} A_{\mu_{1} \cdots \mu_{(n-2)}}^{(n-2)}\right) \\
& +\frac{1}{2} \delta\left(\sqrt{-g} e^{-2(D-1) \alpha \varphi} \mathcal{F}^{\mu \nu}\right)
\end{aligned}
$$

In light of the formula (2.24), the conserved charge associated with the potential (5.17) is defined by

$$
\tilde{\mathcal{Q}}_{z}=\mathcal{Q}_{z}-\frac{n(n-1)}{8 \pi G_{(D)}} \int_{\partial \Sigma} \gamma e^{-2(n-1) \alpha \varphi} \tilde{F}_{(n)}^{\mu \mu_{1} \cdots \mu_{(n-2)} \nu} A_{\mu_{1} \cdots \mu_{(n-2)}}^{(n-2)} d \Sigma_{\mu \nu}
$$

where the charge $\mathcal{Q}_{z}$ is given by Eq. (4.14). The charge $\tilde{\mathcal{Q}}_{z}$ is the angular momentum along the $z$ coordinate in $(D+1)$ dimensions. In analogy with the case of Einstein gravity in the last section, from a $D$-dimensional perspective, $\tilde{\mathcal{Q}}_{z}$ can be thought of as the electric charge associated with the $U(1)$ gauge field $\mathcal{A}_{\mu}$ in the lower-dimensional reduced theory described by the Lagrangian (5.4).

It is worth noting that we have assumed that the higher-dimensional geometry allows a compactified dimension to exist so that its metric ansatz can be decomposed as the form in 
Eq. (4.2), in order to get the property that the off-shell generalized ADT charges remain the same at mathematical level before and after Kaluza-Klein dimensional reduction in the frame work of the Einstein gravity theory and the gravity theories with a single $n$-form field strength. All the results demonstrate that the consistent dimensional reduction can yields consistent conserved charges. Due to this, one is able to simplify the calculations of the conserved charges via performing dimensional reduction or lifting. Generally speaking, the higher-dimensional gravity theories consist of less fields and the Lagrangian describing these theories is more compact. Accordingly, the off-shell ADT potential for the Lagrangian is simpler. Consequently, to simplify calculations, the conserved charges for the lowerdimensional theories can be evaluated in higher dimensions by performing dimensional lifting. This will be illustrated in the next section.

\section{An example: conserved charges of Kaluza-Klein black holes}

As an application of the general derivation in section 4, we shall explicitly evaluate the mass and angular momentum of five-dimensional rotating Kaluza-Klein black holes found in [45] in this section. These black holes are the exact solution of pure Einstein gravity in five dimensions, described by the Einstein-Hilbert Lagrangian $\hat{\mathcal{L}}_{(5 D)}=\sqrt{-\hat{g}} \hat{R}$. Under KaluzaKlein dimensional reduction from five dimensions to four dimensions, the Kaluza-Klein black holes can be seen as the rotating black holes with both electric and magnetic charges in the four-dimensional Einstein-Maxwell-dilaton theory with a particular dilaton coupling. According to the lower-dimensional Lagrangian (4.4), the four-dimensional Lagrangian for this theory takes the form

$$
\mathcal{L}_{(4 D)}=\sqrt{-g}\left(R-\frac{1}{2} \nabla^{\mu} \varphi \nabla_{\mu} \varphi-\frac{1}{4} e^{-\sqrt{3} \varphi} \mathcal{F}^{2}\right) .
$$

Within the context of the five-dimensional Einstein gravity, the rotating Kaluza-Klein black hole solution is read off as [45]

$$
\begin{aligned}
d \hat{s}_{(5)}^{2}= & \frac{H_{2}}{H_{1}}\left(d z+A_{(4 d)}\right)^{2}-\frac{H_{3}}{H_{2}}\left(d t+\left[2 J(r-m)+m^{2} a(p q)^{\frac{1}{2}}\right] \sin ^{2} \theta \frac{d \phi}{H_{3}}\right)^{2} \\
& +H_{1}\left(\frac{d r^{2}}{\Delta(r)}+d \theta^{2}+\Delta(r) \sin ^{2} \theta \frac{d \phi^{2}}{H_{3}}\right)
\end{aligned}
$$

where the compactified coordinate $z \sim z+2 \pi L$, the functions $\Delta(r), H_{1}, H_{2}$ and $H_{3}$ are 
given by

$$
\begin{aligned}
\Delta(r) & =r^{2}+m^{2} a^{2}-2 m r, \quad H_{3}=\Delta(r)-m^{2} a^{2} \sin ^{2} \theta, \\
H_{1} & =H_{3}+p(r-m)+\frac{2}{a} \sqrt{(p / q)}\left(J+a^{2} P_{m} Q_{e} \cos \theta\right), \\
H_{2} & =H_{3}+q(r-m)+\frac{2}{a} \sqrt{(q / p)}\left(J-a^{2} P_{m} Q_{e} \cos \theta\right),
\end{aligned}
$$

respectively, while the Kaluza-Klein vector $A_{(4 d)}$ reads

$$
\begin{aligned}
A_{(4 d)}= & -\left[Q_{e}(2 r+p-2 m)-a P_{m} \sqrt{\left(q^{3} / p\right)} \cos \theta\right] \frac{d t}{H_{2}} \\
& -\left\{2 P_{m}\left(H_{2}+m^{2} a^{2} \sin ^{2} \theta\right) \cos \theta\right. \\
& \left.-(p q)^{-\frac{1}{2}} Q_{e}\left[p^{2} r-m p(p-2 m)+4 q P_{m}^{2}\right] a \sin ^{2} \theta\right\} \frac{d \phi}{H_{2}} .
\end{aligned}
$$

In Eqs. (6.2), (6.3) and (6.4), the parameters $(a, m, p, q)$ are integral constants, while the parameters $\left(J, P_{m}, Q_{e}\right)$ are presented by

$$
\begin{aligned}
J & =\frac{\sqrt{p q}\left(p q+4 m^{2}\right)}{4(p+q)} a \\
P_{m} & =\frac{1}{2} \sqrt{\frac{p\left(p^{2}-4 m^{2}\right)}{p+q}}, \quad Q_{e}=\frac{1}{2} \sqrt{\frac{q\left(q^{2}-4 m^{2}\right)}{p+q}},
\end{aligned}
$$

where $P_{m}$ is the magnetic charge for the reduced four-dimensional black hole, and it will be shown that the parameters $J$ and $Q_{e}$ denote the angular momentum and electric charge respectively. It is worth noting that the parameter $a$ in the metric given in [45] is substituted by $m a$ in the present metric (6.2), like in [46].

By performing dimensional reduction to the rotating black hole solution (6.2) from five dimensions to four dimensions, we get the four-dimensional black hole solution

$$
\begin{aligned}
d s_{(4)}^{2}= & -\frac{H_{3}}{\sqrt{H_{1} H_{2}}}\left(d t+\left[2 J(r-m)+m^{2} a \sqrt{p q}\right] \sin ^{2} \theta \frac{d \phi}{H_{3}}\right)^{2} \\
& +\sqrt{H_{1} H_{2}}\left(\frac{d r^{2}}{\Delta(r)}+d \theta^{2}+\frac{\Delta(r)}{H_{3}} \sin ^{2} \theta d \phi^{2}\right), \\
e^{\varphi / \sqrt{3}}= & \sqrt{H_{1} / H_{2}}, \quad \mathcal{A}=A_{(4 d)},
\end{aligned}
$$

which is an exact solution corresponding to the Lagrangian (6.1).

In terms of the five-dimensional metric ansatz (6.2), we now compute the mass and angular momentum of the Kaluza-Klein black holes. The fluctuation of the gravitational 
field is determined by the infinitesimal change of the parameters $(m, a, p, q)$, that is,

$$
m \rightarrow m+d m, \quad a \rightarrow a+d a, \quad p \rightarrow p+d p, \quad q \rightarrow q+d q
$$

The Killing vectors associated with mass and the angular momentum along the $\phi$ direction are $\hat{\xi}_{(t)}^{\hat{\mu}}=(-1,0,0,0,0)$ and $\hat{\xi}_{(\phi)}^{\hat{\mu}}=(0,0,0,1,0)$ respectively. With the help of Eq. (4.5), the $(t, r)$ components of the off-shell ADT potentials corresponding to these Killing vectors are given by

$$
\begin{aligned}
& \sqrt{-\hat{g}} \hat{Q}_{(K K)}^{t r}\left(\hat{\xi}_{(t)}\right)=d(p)+d(q)+\mathcal{O}\left(\frac{1}{r}\right), \\
& \sqrt{-\hat{g}} \hat{Q}_{(K K)}^{t r}\left(\hat{\xi}_{(\phi)}\right)=4 d(J)+\mathcal{O}\left(\frac{1}{r}\right) .
\end{aligned}
$$

By making use of the formula (2.24) for the conserved charges, the mass $M$ and the angular memontum $J_{\phi}$ reads

$$
M=\frac{p+q}{4 G_{4}}, \quad J_{\phi}=\frac{J}{G_{4}} .
$$

Here the mass $M$ is identified with the ADM mass but different from the Komar mass, which is $M_{\text {Komar }}=q /\left(4 G_{4}\right)$.

On the other hand, it is completely feasible for us to evaluate the mass and the angular momentum along the $\phi$ coordinate in terms of the four-dimensional reduced metric (6.6). They coincide with the ones obtained in five dimensions respectively. This verifies the frontal conclusion that the off-shell generalized ADT charges are invariant under Kaluza-Klein dimensional reduction. However, in the four-dimensional case, apart from the gravitational field, the gauge field and scalar field have to be taken into consideration, so the calculations of the conserved charges are more involved. Moreover, one can test that the angular momentum along the compactified direction $z$ is equal to the electric charge $Q_{e}$ of the four-dimensional black hole (6.6).

\section{Conclusions and discussions}

In the present work, to provide another novel understanding of the conserved charges for gravity theories, we investigate the conserved charges of generally diffeomorphism invariant gravity theories with various matter fields, particularly the ones with scalar fields and $p$ form potentials, through the off-shell generalized ADT formalism, as well as the properties of the conserved charges under Kaluza-Klein dimensional reduction on a circle $S^{1}$. 
First, we construct an off-shell generalized ADT current (2.14) within the framework of the generally diffeomorphism invariant gravity theories described by the generic Lagrangian (2.1) through the linear combination of the two currents in Eqs. (2.11) and (2.13). The former arises from the variation of equation (2.9), which is the total divergence for the contraction of the vector $\zeta_{\nu}$ with the tensor $\mathcal{E}^{\mu \nu}$ made up of expressions of the field equations, while the latter is deduced from the Lie derivative for the variation equation (2.2) of the Lagrangian with respect to the Killing vector. The newly constructed ADT current, containing the term $\mathcal{L}_{\xi} \Theta^{\mu}\left(\delta g, \delta \psi^{(r)}\right)$ and the terms with the variation of the Killing vector, formally differs from those presented in [9, 10, 11]. However, in essence, all the off-shell ADT currents are equivalent since $\mathcal{L}_{\xi} \Theta^{\mu}\left(\delta g, \delta \psi^{(r)}\right)=0$ holds for generally diffeomorphism gravity theories when $\delta \xi^{\mu}=0$, while the variation of the Killing vectors associated with mass and angular momentum always disappears. The merits of the ADT current (2.14) are that the procedure to derive this current becomes simple and it makes a natural and practical construction for derivation of its corresponding potential. Moving on to derive the off-shell ADT potential (2.20), we further present the formula (2.24) for the conserved charges associated with the Lagrangian (2.1).

Second, we present a generic Lagrangian (3.1) that describes a wide range of gravity theories consisting of a gravitational field, multiple scalar fields and $p$-form potentials, thanks to the $L_{M}$ term of this Lagrangian, which possesses the two general structures in Eqs. (3.2) and (3.3) so that it incorporates a large range of terms made up of scalar fields and $p$-form potentials for the Lagrangians in the context of various gravity theories with these matter fields. Making use of the off-shell generalized ADT formalism, we derive the off-shell Noether current (3.12) and ADT potential (3.17) associated with the Lagrangian (3.1). Furthermore, substituting the potential (3.17) into the formula (2.24), one can obtain the formulation of conserved charges for this Lagrangian. As indicated before, the Lagrangian (3.1) is very generic. Hence, the formulation of conserved charges is applicable to supergravity theories, the Einstein-Maxwell-dilaton theory, the low-energy effective field theory of heterotic string theory and so on.

Third, we investigate the behaviour of the conserved charges for the theory of Einstein gravity as well as the gravity theory with a single $p$-form potential and multiple scalar fields in arbitrary dimensions by performing the Kaluza-Klein dimensional reduction along a compactified direction. To both the two types of gravity theories, as demonstrated by Eqs. (4.11) and (5.16), the $(\mu, \nu)$ components of the $(D+1)$-dimensional off-shell ADT potential 
multiplied by the factor $\sqrt{-\hat{g}}$ coincide with those multiplied by the factor $\sqrt{-g}$ in $D$ dimensions. This directly leads to the conclusion that the conserved charges defined in terms of the off-shell generalized ADT formalism are invariant under Kaluza-Klein reduction on a circle. On the other hand, the charges given by Eqs. (4.14) and (5.18) further indicate that the angular momentum along the compactified direction in $(D+1)$ dimensions is just the electric charge with respect to the Kaluza-Klein vector $\mathcal{A}_{\mu}$ in the $D$-dimensional reduced theory. What is more, it has been shown that the off-shell generalized ADT method is essentially equivalent with the LIW and BBC methods. As a result, the formulation of conserved charges defined on the basis of both the two methods naturally exhibit the properties of the off-shell ADT formalism under Kaluza-Klein reduction. To illustrate our calculations, we explicitly evaluate the mass and angular momentum of the five-dimensional rotating Kaluza-Klein black hole (6.2). These conserved charges coincide with their corresponding ones for the reduced four-dimensional black hole (6.6).

Besides, there are several issues deserving to be discussed and investigated in the future work. We first take into consideration of the potential $\check{\mathcal{Q}}^{\mu \nu}$ given in Eq. (2.23), which arises from the linear combination of the current $\mathcal{J}_{[1]}^{\mu}$ with the one $\mathcal{J}_{[2]}^{\mu}$ and depends on a generic constant $k$. In the case where $k \neq 0$ and all the fields are off-shell, further investigation is demanded to clarify whether this potential can be employed to present well-defined and meaningful conserved charges of gravity theories, as well as to understand the meaning of these conserved charges if it is possible. Next, the formula (2.24) endowed with the potential (3.17) can be employed to derive the first law of thermodynamics for black holes with $p$-form gauge fields and scalar fields.

Apart from the above two issues, as mentioned before, we have analyzed the behaviour of the conserved charges for the Lagrangian (5.1) that includes a sole $p$-form potential and multiple scalar fields under Kaluza-Klein reduction. The results demonstrate that the conserved charges for this Lagrangian are invariant before and after dimensional reduction on a circle. As a matter of course, the analysis should be able to apply to the more generic Lagrangian $\mathcal{L}_{M}$ with the function $L_{M}$ given by Eq. (3.4), which contains a number of $p$-form potentials. Unfortunately, the calculations are very complex. However, we have checked several other special cases of $\mathcal{L}_{M}$ and found that the conserved charges are indeed invariant. As a consequence, we suppose that the conserved charges defined in terms of the off-shell generalized ADT formalism are unchanged undergoing Kaluza-Klein reduction along a compactified direction even for the Lagrangian (3.4) that consists of a lot of $p$-form 
potentials as well as a number of scalar fields. Once this guess holds, one can further see that the conserved charges associated with this Lagrangian are still unchanged under a reduction from $(D+d)$ dimensions to $D$ dimensions on the $d$-torus $T^{d}=S^{1} \times \cdots \times S^{1}$ because of the generality of the Lagrangian. Another interesting issue is to prove that the conserved charges via the off-shell generalized ADT formulation is invariant under KaluzaKlein reduction in the framework of generic covariant gravity theories involving arbitrary matter fields and higher curvature terms.

\section{Acknowledgments}

This work was supported by the Natural Science Foundation of China under Grant No. 11505036 and No. 11275157. It was also partially supported by the Technology Department of Guizhou province Fund under Grant No. (2016)1104 and the Guizhou province science and technology innovation talent team [Grant No. (2015)4015].

\section{A The off-shell Noether currents and potentials of the La- grangians $\mathcal{L}_{M}$ and $\mathcal{L}_{\phi}$}

In this appendix, we present detailed calculations for the derivations of the off-shell Noether currents and potentials associated with the Lagrangians $\mathcal{L}_{M}=\sqrt{-g} L_{M}$ and $\mathcal{L}_{\phi}=\sqrt{-g} L_{\phi}$ given in Eq. (3.1). It has been shown in section 3 that $L_{M}$ is assumed to take three types of general forms, i.e., the ones in Eqs. (3.2), (3.3) and (3.4). Accordingly, our analysis associated with the Lagrangian $\mathcal{L}_{M}$ is divided into three parts.

We first take into account the Noether current and potential for the Lagrangian $\mathcal{L}_{M}$. When $L_{M}$ takes the form as the one in Eq. (3.2), the variation of the Lagrangian $\mathcal{L}_{M}$ is read off as

$$
\begin{aligned}
\delta \mathcal{L}_{M}= & \sqrt{-g}\left[\mathcal{E}_{(M) \mu \nu}^{(g r)} \delta g^{\mu \nu}+\sum_{k=1}^{m} \mathcal{E}_{(k)}^{(\phi)} \delta \phi^{(k)}+\sum_{p=1}^{n-1} \mathcal{E}_{(p)}^{(A) \mu_{1} \cdots \mu_{p}} \delta A_{(p) \mu_{1} \cdots \mu_{p}}\right. \\
& \left.+\nabla_{\mu} \Theta_{(M)}^{\mu}\left(\delta g, \delta \phi^{(k)}, \delta A_{(p)}\right)\right] .
\end{aligned}
$$

Here the expressions of the field equations for the gravitational field, scalar fields and the 
$p$-form potentials, as well as the surface term are given by

$$
\begin{aligned}
\mathcal{E}_{(M) \mu \nu}^{(g r)} & =\frac{\partial L_{M}}{\partial g^{\mu \nu}}-\frac{1}{2} g_{\mu \nu} L_{M}, \quad \mathcal{E}_{(k)}^{(\phi)}=\frac{\partial L_{M}}{\partial \phi^{(k)}}, \\
\mathcal{E}_{(p)}^{(A) \mu_{1} \cdots \mu_{p}} & =B_{(p)}^{\mu_{1} \cdots \mu_{p}}-(p+1) \nabla_{\mu} U_{(p+1)}^{\mu \mu_{1} \cdots \mu_{p}}, \\
\Theta_{(M)}^{\mu} & =\sum_{p=1}^{n-1}(p+1) U_{(p+1)}^{\mu \mu_{1} \cdots \mu_{p}} \delta A_{(p) \mu_{1} \cdots \mu_{p}},
\end{aligned}
$$

respectively, where the two totally antisymmetric tensors $B_{(p)}^{\mu_{1} \cdots \mu_{p}}$ and $U_{(p+1)}^{\mu_{1} \cdots \mu_{(p+1)}}$ are defined through

$$
\begin{aligned}
B_{(p)}^{\mu_{1} \cdots \mu_{p}} & =\frac{\partial L_{M}}{\partial A_{(p) \mu_{1} \cdots \mu_{p}}}, \\
U_{(p+1)}^{\mu_{1} \cdots \mu_{(p+1)}} & =\frac{\partial L_{M}}{\partial F_{(p+1) \mu_{1} \cdots \mu_{(p+1)}}},
\end{aligned}
$$

respectively, and it is proved that the symmetric tensor $\partial L_{M} / \partial g^{\mu \nu}$ satisfies

$$
\frac{\partial L_{M}}{\partial g^{\mu \nu}}=\frac{1}{2} \sum_{p=1}^{n-1}\left[p B_{(p) \mu}^{\mu_{1} \cdots \mu_{(p-1)}} A_{(p) \nu \mu_{1} \cdots \mu_{(p-1)}}+(p+1) U_{(p+1) \mu}{ }^{\mu_{1} \cdots \mu_{p}} F_{(p+1) \nu \mu_{1} \cdots \mu_{p}}\right] .
$$

The Lie derivative of $L_{M}$ along the $\zeta^{\mu}$ vector is

$$
\begin{aligned}
\zeta^{\nu} \nabla_{\nu} L_{M}= & \sum_{k=1}^{m} \mathcal{E}_{(k)}^{(\phi)} \zeta^{\nu} \nabla_{\nu} \phi^{(k)}+\sum_{p=1}^{n-1} B_{(p)}^{\mu_{1} \cdots \mu_{p}} \zeta^{\nu} \nabla_{\nu} A_{(p) \mu_{1} \cdots \mu_{p}} \\
& +\sum_{p=1}^{n-1} U_{(p+1)}^{\mu_{1} \cdots \mu_{(p+1)}} \zeta^{\nu} \nabla_{\nu} F_{(p+1) \mu_{1} \cdots \mu_{(p+1)}} .
\end{aligned}
$$

Substituting both the following equations

$$
\begin{aligned}
B_{(p)}^{\mu_{1} \cdots \mu_{p}} \zeta^{\nu} \nabla_{\nu} A_{(p) \mu_{1} \cdots \mu_{p}}= & B_{(p)}^{\mu_{1} \cdots \mu_{p}} \zeta^{\nu} F_{(p+1) \nu \mu_{1} \cdots \mu_{p}} \\
& -p\left(\nabla_{\mu} B_{(p)}^{\mu \mu_{1} \cdots \mu_{(p-1)}}\right) \zeta^{\nu} A_{(p) \nu \mu_{1} \cdots \mu_{(p-1)}} \\
& +p \zeta^{\nu} \nabla_{\mu}\left(B_{(p)}^{\mu \mu_{1} \cdots \mu_{(p-1)}} A_{(p) \nu \mu_{1} \cdots \mu_{(p-1)}}\right) \\
U_{(p+1)}^{\mu_{1} \cdots \mu_{(p+1)}} \zeta^{\nu} \nabla_{\nu} F_{(p+1) \mu_{1} \cdots \mu_{(p+1)}}= & (p+1) U_{(p+1)}^{\mu \mu_{1} \cdots \mu_{p}} \zeta^{\nu} \nabla_{\mu} F_{(p+1) \nu \mu_{1} \cdots \mu_{p}}
\end{aligned}
$$

into Eq. (A.5), one arrives at the following

$$
\begin{aligned}
\zeta^{\nu} \nabla_{\nu} L_{M}= & \sum_{k=1}^{m} \mathcal{E}_{(k)}^{(\phi)} \zeta^{\nu} \nabla_{\nu} \phi^{(k)}+\sum_{p=1}^{n-1} \mathcal{E}_{(p)}^{(A) \mu_{1} \cdots \mu_{p}} \zeta^{\nu} F_{(p+1) \nu \mu_{1} \cdots \mu_{p}} \\
& -\sum_{p=1}^{n-1} p\left(\nabla_{\mu} \mathcal{E}_{(p)}^{(A) \mu \mu_{1} \cdots \mu_{(p-1)}}\right) \zeta^{\nu} A_{(p) \nu \mu_{1} \cdots \mu_{(p-1)}}+2 \zeta^{\nu} \nabla^{\mu}\left(\frac{\partial L_{M}}{\partial g^{\mu \nu}}\right) .
\end{aligned}
$$


On the other hand, for a $p$-form potential $A_{(p)}$, its Lie derivative along the vector $\zeta^{\mu}$ can be defined as

$$
\mathcal{L}_{\zeta} A_{(p) \mu_{1} \cdots \mu_{p}}=\zeta^{\nu} F_{(p+1) \nu \mu_{1} \cdots \mu_{p}}-\sum_{k=1}^{p}(-1)^{k} \nabla_{\mu_{k}}\left(\zeta^{\nu} A_{(p) \nu \mu_{1} \cdots \mu_{(k-1)} \mu_{(k+1)} \cdots \mu_{p}}\right) .
$$

Making use of this definition, we have

$$
\begin{aligned}
\mathcal{E}_{(p) \mu_{1} \cdots \mu_{p}}^{(A)} \mathcal{L}_{\zeta} A_{(p) \mu_{1} \cdots \mu_{p}}= & \mathcal{E}_{(p)}^{(A) \mu_{1} \cdots \mu_{p}} \zeta^{\nu} F_{(p+1) \nu \mu_{1} \cdots \mu_{p}} \\
& -p\left(\nabla_{\mu} \mathcal{E}_{(p) \mu \mu_{1} \cdots \mu_{(p-1)}}^{(A)}\right) \zeta^{\nu} A_{(p) \nu \mu_{1} \cdots \mu_{(p-1)}} \\
& +p \nabla_{\mu}\left(\mathcal{E}_{(p)}^{(A) \mu \mu_{1} \cdots \mu_{(p-1)}} \zeta^{\nu} A_{(p) \nu \mu_{1} \cdots \mu_{(p-1)}}\right)
\end{aligned}
$$

With help of Eqs. (A.7) and (A.9), one can obtain the identity

$$
\begin{aligned}
2 \nabla_{\mu}\left(\mathcal{E}_{(M)}^{\mu \nu} \zeta_{\nu}\right)= & 2 \mathcal{E}_{(M)}^{(g r) \mu \nu} \nabla_{\mu} \zeta_{\nu}-\sum_{k=1}^{m} \mathcal{E}_{(k)}^{(\phi)} \zeta^{\nu} \nabla_{\nu} \phi^{(k)} \\
& -\sum_{p=1}^{n-1} \mathcal{E}_{(p)}^{(A) \mu_{1} \cdots \mu_{p}} \mathcal{L}_{\zeta} A_{(p) \mu_{1} \cdots \mu_{p}}
\end{aligned}
$$

where the $\mathcal{E}_{(M)}^{\mu \nu}$ tensor is read off as

$$
\mathcal{E}_{(M)}^{\mu \nu}=\mathcal{E}_{(M)}^{(g r) \mu \nu}-\frac{1}{2} \sum_{p=1}^{n-1} p \mathcal{E}_{(p)}^{(A) \mu \mu_{1} \cdots \mu_{(p-1)}} A_{(p) \mu_{1} \cdots \mu_{(p-1)}}^{\nu} .
$$

It is shown in Eq. (A.11) that the non-symmetric 2-rank tensor $\mathcal{E}_{(M)}^{\mu \nu}$ is only the combination for the expressions of the field equations $\mathcal{E}_{(M)}^{(g r) \mu \nu}$ and $\mathcal{E}_{(p)}^{(A) \mu_{1} \cdots \mu_{p}}$, which are associated with the gravitational field $g_{\mu \nu}$ and the gauge fields $A_{(p)}$ respectively, while the contribution from the scalar fields $\phi^{(k)}$ is absent. Equation (A.10) tests the identity (2.3) for generic covariant theories. In particular, when the vector $\zeta^{\mu}$ becomes the Killing vector $\xi^{\mu}$ that satisfies the conditions in Eq. (2.8), Eq. (A.10) yields that the contraction between the tensor $\mathcal{E}_{(M)}^{\mu \nu}$ and the Killing vector is divergence-free.

Following the procedure to derive the general off-shell Noether current (2.6) in section 2. one can further define an off-shell Noether current $J_{(M)}^{\mu}$ with respect to the Lagrangian $\mathcal{L}_{M}$ in terms of Eqs. (A.1) and (A.10) by

$$
\begin{aligned}
J_{(M)}^{\mu} & =2 \mathcal{E}_{(M)}^{\mu \nu} \zeta_{\nu}+\zeta^{\mu} L_{M}-\Theta_{(M)}^{\mu}\left(\mathcal{L}_{\zeta} A_{(p)}\right) \\
& =\nabla_{\nu} K_{(M)}^{\mu \nu}
\end{aligned}
$$


where the off-shell Noether potential $K_{(M)}^{\mu \nu}$ is presented by

$$
K_{(M)}^{\mu \nu}=-\sum_{p=1}^{n-1} p(p+1) U_{(p+1)}^{\mu \nu \mu_{1} \cdots \mu_{(p-1)}} \zeta^{\sigma} A_{(p) \sigma \mu_{1} \cdots \mu_{(p-1)}}
$$

which demonstrates that only the field strengths $\left(F_{\left(q_{1}\right)}, \cdots, F_{\left(q_{J}\right)}\right)$ in $L_{M}$ are associated with the off-shell Noether potential, while all the potentials $\left(A_{\left(p_{1}\right)}, \cdots, A_{\left(p_{I}\right)}\right)$ make no contribution to the potential.

On the other hand, in the case where $L_{M}$ takes the same form as the one in Eq. (3.3), one only needs to postulate that the Levi-Civita tensor density $\bar{\epsilon}_{\mu_{1} \cdots \mu_{D}}$ is a $D$-form "potential" $A_{(D) \mu_{1} \cdots \mu_{D}}=\bar{\epsilon}_{\mu_{1} \cdots \mu_{D}}$. Under such an assumption, one obtains that $F_{(D+1)}=d A_{(D)}=0$, $\nabla_{\mu}\left(\sqrt{-g} A_{(D) \mu_{1} \cdots \mu_{D}}\right)=0$ and $B_{(D)}^{\mu_{1} \cdots \mu_{D}}=0$ because of the disappearance of variation for the $A_{(D)}$ potential. Due to these facts, one can further observe that all the results with respect to $L_{M}$ described by Eq. (3.2) still hold in the case where $L_{M}$ possesses the form in Eq. (3.3). This means that all the results are identified for both the forms of $L_{M}$.

What is more, in the case where $L_{M}$ takes the form given in Eq. (3.4), that is $L_{M}=\mathbb{L}_{M}$, all the results are equivalent with those for $L_{M}$ with the generic form in Eq. (3.2). Therefore, here we do not plan to give detailed derivations but directly present the surface term $\Theta_{\left(\mathbb{L}_{M}\right)}^{\mu}$ and the off-shell Noether potential $K_{\left(\mathbb{L}_{M}\right)}^{\mu \nu}$. The former, deduced from the surface term $\Theta_{(M)}^{\mu}$ in Eq. (A.2), has the form

$$
\begin{aligned}
\Theta_{\left(\mathbb{L}_{M}\right)}^{\mu}= & \sum_{c=1}^{t} q_{c} \mathbb{U}_{\left(q_{c}\right)}^{\mu \mu_{1} \cdots \mu_{\left(q_{c}-1\right)}} \delta A_{\left(q_{c}-1\right) \mu_{1} \cdots \mu_{\left(q_{c}-1\right)}} \\
& +\sum_{c=1}^{j} \tilde{q}_{c} \mathbb{U}_{\left(\tilde{q}_{c}\right)}^{\mu \mu_{1} \cdots \mu_{\left(\tilde{q}_{c}-1\right)}} \delta A_{\left(\tilde{q}_{c}-1\right) \mu_{1} \cdots \mu_{\left(\tilde{q}_{c}-1\right)}},
\end{aligned}
$$

where the totally antisymmetric $q_{c}\left(\tilde{q}_{c}\right)$-rank tensors $\mathbb{U}_{\left(q_{c}\right)}^{\mu_{1} \cdots \mu_{q_{c}}}$ and $\mathbb{U}_{\left(\tilde{q}_{c}\right)}^{\mu_{1} \cdots \mu_{\tilde{q}_{c}}}$ are defined by the second equation in Eq. (A.3), namely, $\mathbb{U}_{\left(q_{c} / \tilde{q}_{c}\right)}=\partial \mathbb{L}_{M} / \partial F_{\left(q_{c} / \tilde{q}_{c}\right)}$. However, it is worth noting that all the field strengths $F_{\left(q_{c} / \tilde{q}_{c}\right)}$ are treated as independent variables when the second equation in Eq. (A.3) is applied to get $\mathbb{U}_{\left(q_{c} / \tilde{q}_{c}\right)}$. By following this, when $1 \leq c \leq t$, the tensor $\mathbb{U}_{\left(q_{c}\right)}$ reads

$$
\begin{aligned}
\mathbb{U}_{\left(q_{c}\right)}^{\mu_{1} \cdots \mu_{q_{c}}} & =W \mathbb{H}_{[c] \mu_{\left(q_{c}+1\right)} \cdots \mu_{N}} Y_{(N)}^{\mu_{1} \cdots \mu_{N}} \\
\mathbb{H}_{[c] \mu_{\left(q_{c}+1\right)} \cdots \mu_{N}} & =\left(F_{\left(q_{1}\right)} \cdots F_{\left(q_{(c-1)}\right)} F_{\left(q_{(c+1)}\right)} \cdots F_{\left(q_{t}\right)} A_{\left(p_{1}\right)} \cdots A_{\left(p_{s}\right)}\right)_{\left[\mu_{\left(q_{c}+1\right)} \cdots \mu_{N}\right]}
\end{aligned}
$$


In addition, when $1 \leq c \leq j$, the tensor $\mathbb{U}_{\left(\tilde{q}_{c}\right)}$ is given by

$$
\begin{aligned}
\mathbb{U}_{\left(\tilde{q}_{c}\right)}^{\mu_{1} \cdots \mu_{\tilde{q}_{c}}} & =W H_{(N)}^{\mu_{1} \cdots \mu_{N}} \mathbb{Y}_{[c] \mu_{\left(\tilde{q}_{c}+1\right)} \cdots \mu_{N}} \\
\mathbb{Y}_{[c] \mu_{\left(\tilde{q}_{c}+1\right)} \cdots \mu_{N}} & =\left(F_{\left(\tilde{q}_{1}\right)} \cdots F_{\left(\tilde{q}_{(c-1)}\right)} F_{\left(\tilde{q}_{(c+1)}\right)} \cdots F_{\left(\tilde{q}_{j}\right)} A_{\left(\tilde{p}_{1}\right)} \cdots A_{\left(\tilde{p}_{i}\right)}\right)_{\left[\mu_{\left(\tilde{q}_{c}+1\right)} \cdots \mu_{N}\right]}
\end{aligned}
$$

Starting out with Eq. (A.13), we derive the off-shell Noether potential associated with $\mathbb{L}_{M}$, that is,

$$
\begin{aligned}
K_{\left(\mathbb{L}_{M}\right)}^{\mu \nu}= & -\sum_{c=1}^{t} q_{c}\left(q_{c}-1\right) \mathbb{U}_{\left(q_{c}\right)}^{\mu \nu \mu_{1} \cdots \mu_{\left(q_{c}-2\right)}} \zeta^{\sigma} A_{\left(q_{c}-1\right) \sigma \mu_{1} \cdots \mu_{\left(q_{c}-2\right)}} \\
& -\sum_{c=1}^{j} \tilde{q}_{c}\left(\tilde{q}_{c}-1\right) \mathbb{U}_{\left(\tilde{q}_{c}\right)}^{\mu \nu \mu_{1} \cdots \mu_{\left(\tilde{q}_{c}-2\right)}} \zeta^{\sigma} A_{\left(\tilde{q}_{c}-1\right) \sigma \mu_{1} \cdots \mu_{\left(\tilde{q}_{c}-2\right)}} .
\end{aligned}
$$

Here the Noether potential $K_{\left(\mathbb{L}_{M}\right)}^{\mu \nu}$, as well as the surface term in Eq. (A.14), is also applicable to the case where $L_{M}$ has the same form as the one in Eq. (3.3). For example, in such a case, if it is supposed that $Y_{\mu_{1} \cdots \mu_{D}}^{(D)}=\bar{\epsilon}_{\mu_{1} \cdots \mu_{D}}$, one finds that the surface term and the Noether potential are just the ones given in Eqs. (A.14) and (A.17) in the absence of the $\mathbb{U}_{\left(\tilde{q}_{c}\right)}$ term, respectively.

In the following of this appendix, we calculate the off-shell Noether current and potential of the Lagrangian $\mathcal{L}_{\phi}$. The variation of the Lagrangian yields

$$
\delta \mathcal{L}_{\phi}=\sqrt{-g}\left[\mathcal{E}_{(\phi) \mu \nu}^{(g r)} \delta g^{\mu \nu}+\sum_{k=1}^{m} \tilde{\mathcal{E}}_{(k)}^{(\phi)} \delta \phi^{(k)}+\nabla_{\mu} \Theta_{(\phi)}^{\mu}\left(\delta \phi^{(k)}\right)\right]
$$

where the expressions for the field equations $\mathcal{E}_{(\phi) \mu \nu}^{(g r)}$ and $\tilde{\mathcal{E}}_{(k)}^{(\phi)}$ for the gravitational field and scalar fields respectively, are given by

$$
\begin{aligned}
\mathcal{E}_{(\phi) \mu \nu}^{(g r)} & =\sum_{i, j=1}^{m} X_{i j} \nabla^{\mu} \phi^{(i)} \nabla_{\mu} \phi^{(j)}-\frac{1}{2} g_{\mu \nu} L_{\phi} \\
\tilde{\mathcal{E}}_{(k)}^{(\phi)} & =-2 \sum_{i=1}^{m} \nabla_{\mu}\left(X_{i k} \nabla^{\mu} \phi^{(i)}\right)+\sum_{i, j=1}^{m} \frac{\partial X_{i j}}{\partial \phi^{(k)}} \nabla^{\mu} \phi^{(i)} \nabla_{\mu} \phi^{(j)}+\frac{\partial V}{\partial \phi^{(k)}},
\end{aligned}
$$

and the surface term $\Theta_{(\phi)}^{\mu}$ is read off as

$$
\Theta_{(\phi)}^{\mu}=2 \sum_{i, j=1}^{m} X_{i j}\left(\nabla^{\mu} \phi^{(i)}\right) \delta \phi^{(j)}
$$


For the expressions for the equations of motion $\mathcal{E}_{(\phi) \mu \nu}^{(g r)}$ and $\tilde{\mathcal{E}}_{(k)}^{(\phi)}$, one is able to prove that they satisfy the following identify

$$
2 \nabla_{\mu}\left(\mathcal{E}_{(\phi)}^{\mu \nu} \zeta_{\nu}\right)=2 \mathcal{E}_{(\phi)}^{(g r) \mu \nu} \nabla_{\mu} \zeta_{\nu}-\sum_{k=1}^{m} \tilde{\mathcal{E}}_{(k)}^{(\phi)} \zeta^{\nu} \nabla_{\nu} \phi^{(k)}
$$

where $\mathcal{E}_{(\phi)}^{\mu \nu}=\mathcal{E}_{(\phi)}^{(g r) \mu \nu}$, which contains no expressions of the field equations $\tilde{\mathcal{E}}_{(k)}^{(\phi)}$. This implies that the scalar fields $\phi^{(k)}$ makes no contribution to the divergence term, unlike the case for the gauge fields. Equivalently, Eq. (A.21) can be rewritten as

$$
\nabla_{\mu} \mathcal{E}_{(\phi)}^{(g r) \mu \nu}+\frac{1}{2} \sum_{k=1}^{m} \tilde{\mathcal{E}}_{(k)}^{(\phi)} \nabla^{\nu} \phi^{(k)}=0
$$

which can be regarded as a generalized Bianchi identity for the scalar fields. The same identity appears in the case of Horndeski theory [17].

As before, in terms of the Eqs. (A.18) and (A.21), we get the off-shell Noether current for the Lagrangian $\mathcal{L}_{\phi}$ that has the form

$$
\begin{aligned}
J_{(\phi)}^{\mu} & =2 \mathcal{E}_{(\phi)}^{\mu \nu} \zeta_{\nu}+\zeta^{\mu} L_{\phi}-\Theta_{(\phi)}^{\mu}\left(\mathcal{L}_{\zeta} \phi^{(k)}\right) \\
& =0
\end{aligned}
$$

which yields a vanishing off-shell Noether potential $K_{(\phi)}^{\mu \nu}$, namely,

$$
K_{(\phi)}^{\mu \nu}=0
$$

Nevertheless, in the case of the Horndeski theory, it was shown in [17] that the off-shell Noether potential related to the Lagrangian containing the terms with higher derivatives of the scalar fields is non-zero.

\section{B The $(\mathrm{D}+1)$-dimensional Christoffel symbols and surface term}

In this appendix, we first derive the explicit expressions of the $(D+1)$-dimensional Christoffel symbols $\hat{\Gamma}_{\hat{\mu} \hat{\nu}}^{\hat{\rho}}$ in terms of all the $D$-dimensional fields, such as the gravitational field $g_{\mu \nu}$, the Kaluza-Klein vector $\mathcal{A}_{\mu}$ and the dilaton $\varphi$. Subsequently the Christoffel symbols are used to derive the exact relationship of the higher-dimensional surface term $\hat{\Theta}_{(\hat{R})}^{\hat{\mu}}$ to its lower-dimensional counterpart. 
We now calculate the Christoffel symbols $\hat{\Gamma}_{\hat{\mu} \hat{\nu}}^{\hat{\rho}}$. On basis of the $(D+1)$-dimensional metric ansatz (4.2), the components of the $(D+1)$-dimensional metric tensor $\hat{g}_{\hat{\mu} \hat{\nu}}$ are given in terms of the $D$-dimensional fields by

$$
\hat{g}_{\mu \nu}=e^{2 \alpha \varphi} g_{\mu \nu}+e^{2 \beta \varphi} \mathcal{A}_{\mu} \mathcal{A}_{\nu}, \quad \hat{g}_{\mu z}=e^{2 \beta \varphi} \mathcal{A}_{\mu}, \quad \hat{g}_{z z}=e^{2 \beta \varphi} .
$$

As a result, the inverse $\hat{g}^{\hat{\mu} \hat{\nu}}$ of the metric tensor are presented by

$$
\hat{g}^{\mu \nu}=e^{-2 \alpha \varphi} g^{\mu \nu}, \quad \hat{g}^{\mu z}=-e^{-2 \alpha \varphi} \mathcal{A}^{\mu}, \quad \hat{g}^{z z}=e^{-2 \beta \varphi}+e^{-2 \alpha \varphi} \mathcal{A}_{\mu} \mathcal{A}^{\mu} .
$$

According to the definition for the Christoffel symbols, together with help of the expressions of $\hat{g}_{\hat{\mu} \hat{\nu}}$ and $\hat{g}^{\hat{\mu} \hat{\nu}}$, the components $\hat{\Gamma}_{\mu \nu}^{\rho}$ and $\hat{\Gamma}_{\mu \nu}^{z}$ of the higher-dimensional Christoffel symbols $\hat{\Gamma}_{\hat{\mu} \hat{\nu}}^{\hat{\rho}}$ are given in terms of the lower-dimensional fields by

$$
\begin{aligned}
\hat{\Gamma}_{\mu \nu}^{\rho}= & \Gamma_{\mu \nu}^{\rho}+e^{-2 \alpha \varphi} \delta_{(\mu}^{\rho} \nabla_{\nu)} e^{2 \alpha \varphi}-\frac{1}{2} e^{-2 \alpha \varphi} g_{\mu \nu} \nabla^{\rho} e^{2 \alpha \varphi} \\
& +e^{2(\beta-\alpha) \varphi} \mathcal{A}_{(\mu} \mathcal{F}_{\nu)}{ }^{\rho}-\frac{1}{2} e^{-2 \alpha \varphi} \mathcal{A}_{\mu} \mathcal{A}_{\nu} \nabla^{\rho} e^{2 \beta \varphi} \\
\hat{\Gamma}_{\mu \nu}^{z}= & -\mathcal{A}_{\rho} \hat{\Gamma}_{\mu \nu}^{\rho}+e^{-2 \beta \varphi} \mathcal{A}_{(\mu} \nabla_{\nu)} e^{2 \beta \varphi}+\partial_{(\mu} \mathcal{A}_{\nu)},
\end{aligned}
$$

where the field strength $\mathcal{F}_{\mu \nu}$ is defined through $\mathcal{F}_{\mu \nu}=2 \partial_{[\mu} \mathcal{A}_{\nu]}$, while the other components are read off as

$$
\begin{aligned}
\hat{\Gamma}_{z z}^{\rho} & =-\frac{1}{2} e^{-2 \alpha \varphi} \nabla^{\rho} e^{2 \beta \varphi}, \\
\hat{\Gamma}_{z z}^{z} & =-\mathcal{A}_{\rho} \hat{\Gamma}_{z z}^{\rho}=\frac{1}{2} e^{-2 \alpha \varphi} \mathcal{A}_{\rho} \nabla^{\rho} e^{2 \beta \varphi}, \\
\hat{\Gamma}_{\mu z}^{\rho} & =-\frac{1}{2} e^{-2 \alpha \varphi} \mathcal{A}_{\mu} \nabla^{\rho} e^{2 \beta \varphi}+\frac{1}{2} e^{2(\beta-\alpha) \varphi} \mathcal{F}_{\mu}{ }^{\rho}, \\
\hat{\Gamma}_{\mu z}^{z} & =-\frac{1}{2} e^{2(\beta-\alpha) \varphi} \mathcal{F}_{\mu}{ }^{\rho} \mathcal{A}_{\rho}+\frac{1}{2} e^{-2 \alpha \varphi} \mathcal{A}_{\mu} \mathcal{A}_{\rho} \nabla^{\rho} e^{2 \beta \varphi}+\frac{1}{2} e^{-2 \beta \varphi} \nabla_{\mu} e^{2 \beta \varphi} .
\end{aligned}
$$

Finally, utilizing the above higher-dimensional Christoffel symbols $\hat{\Gamma}_{\hat{\mu} \hat{\nu}}^{\hat{\rho}}$, we evaluate the $(D+1)$-dimensional surface term $\hat{\Theta}_{(\hat{R})}^{\hat{\mu}}$ in terms of all the $D$-dimensional fields. Its $\hat{\mu}=\mu$ components are presented by

$$
\hat{\Theta}_{(\hat{R})}^{\mu}=\hat{\Theta}_{(\delta g)}^{\mu}+\hat{\Theta}_{(\delta \mathcal{A})}^{\mu}+\hat{\Theta}_{(\delta \phi)}^{\mu}
$$

where

$$
\begin{aligned}
\hat{\Theta}_{(\delta g)}^{\mu}= & -e^{-2 \alpha \varphi}\left[\nabla_{\rho} \delta g^{\rho \mu}-\nabla^{\mu}\left(g_{\rho \sigma} \delta g^{\rho \sigma}\right)\right]-(D \alpha+\beta) e^{-2 \alpha \varphi}\left(\nabla_{\nu} \varphi\right) \delta g^{\mu \nu} \\
& +\alpha e^{-2 \alpha \varphi}\left(\nabla^{\mu} \varphi\right) g_{\rho \sigma} \delta g^{\rho \sigma} \\
\hat{\Theta}_{(\delta \mathcal{A})}^{\mu}= & -e^{2(\beta-2 \alpha) \varphi} \mathcal{F}^{\mu \nu} \delta \mathcal{A}_{\nu}
\end{aligned}
$$


and the $\hat{\Theta}_{(\delta \phi)}^{\mu}$ term is read off as

$$
\begin{aligned}
\hat{\Theta}_{(\delta \phi)}^{\mu}= & {[2(D-1) \alpha-\beta]\left(\nabla^{\mu} \varphi\right) \delta e^{-2 \alpha \varphi}+3 \beta e^{2(\beta-\alpha) \varphi}\left(\nabla^{\mu} \varphi\right) \delta e^{-2 \beta \varphi} } \\
& +(D-1) \nabla^{\mu}\left(\delta e^{-2 \alpha \varphi}\right)+e^{2(\beta-\alpha) \varphi} \nabla^{\mu}\left(\delta e^{-2 \beta \varphi}\right) .
\end{aligned}
$$

Making use of the values of the $\alpha$ and $\beta$ constants in Eq. (4.3), we simplify Eq. (B.5) as

$$
\begin{aligned}
\hat{\Theta}_{(\hat{R})}^{\mu}= & e^{-2 \alpha \varphi}\left[\nabla^{\mu}\left(g_{\rho \sigma} \delta g^{\rho \sigma}\right)-\nabla_{\rho} \delta g^{\rho \mu}-\left(\nabla^{\mu} \varphi\right) \delta \varphi\right. \\
& \left.-e^{-2(D-1) \alpha \varphi} \mathcal{F}^{\mu \nu} \delta \mathcal{A}_{\nu}\right]+e^{-2 \alpha \varphi}\left(\nabla^{\mu} e^{-2 \alpha \varphi}\right) \delta e^{2 \alpha \varphi} \\
& +\frac{1}{\sqrt{-g}} \delta\left(\sqrt{-g} \nabla^{\mu} e^{-2 \alpha \varphi}\right) .
\end{aligned}
$$

Here we do not plan to present the $\hat{\Theta}_{(\hat{R})}^{z}$ component since the definition of the conserved charges does not involve this quantity.

\section{The $(\mathrm{D}+1)$-dimensional Killing vector}

In the present appendix, the main goal consists in deriving the Killing vector in $(D+1)$ dimensions on basis of the features of the $D$-dimensional Killing vector, as well as studying the behaviour of the Lie derivative for the gauge fields with respect to the $(D+1)$-dimensional Killing vector.

We first prove that the $(D+1)$-dimensional $\hat{\xi}_{(b)}^{\hat{\mu}}$ vector, defined through

$$
\hat{\xi}_{(b)}^{\hat{\mu}}=\left(\hat{\xi}_{(b)}^{\mu}, \hat{\xi}_{(b)}^{z}\right)=\left(\xi^{\mu}, b\right),
$$

is a Killing vector, where $\xi^{\mu}$ is the $D$-dimensional Killing vector obeying Eq. (4.8) and the parameter $b$ is an arbitrary constant.

In order to prove $\hat{\xi}_{(b)}^{\hat{\mu}}$ is a Killing vector in $(D+1)$-dimensional spacetime, we only need to prove the following Killing equation

$$
\hat{\nabla}^{\hat{\mu}} \hat{\xi}_{(b)}^{\hat{\nu}}+\hat{\nabla}^{\hat{\nu}} \hat{\xi}_{(b)}^{\hat{\mu}}=0
$$

in local coordinates holds for any value of the vector $\hat{\xi}_{(b)}^{\hat{\mu}}$. With help of the Christoffel symbols in Appendix B, we obtain

$$
\begin{aligned}
\hat{\nabla}^{\mu} \hat{\xi}_{(b)}^{\nu}= & e^{-2 \alpha \varphi} \nabla^{\mu} \xi^{\nu}+\frac{1}{2} e^{-4 \alpha \varphi} g^{\mu \nu} \xi^{\sigma} \nabla_{\sigma} e^{2 \alpha \varphi}+\xi^{[\mu} \nabla^{\nu]} e^{-2 \alpha \varphi} \\
& +\frac{1}{2} e^{2(\beta-2 \alpha) \varphi}\left(b+\mathcal{A}_{\sigma} \xi^{\sigma}\right) \mathcal{F}^{\mu \nu}
\end{aligned}
$$


which yields

$$
\begin{aligned}
\hat{\nabla}^{\mu} \hat{\xi}_{(b)}^{\nu}+\hat{\nabla}^{\nu} \hat{\xi}_{(b)}^{\mu} & =-e^{-2 \alpha \varphi} \mathcal{L}_{\xi} g^{\mu \nu}-g^{\mu \nu} \mathcal{L}_{\xi} e^{-2 \alpha \varphi} \\
& =0=-\mathcal{L}_{\xi} \hat{g}^{\mu \nu} .
\end{aligned}
$$

The above equation implies that the $(\mu, \nu)$ components of Eq. (C.2) holds.

When $\hat{\mu}=\mu$ and $\hat{\nu}=z$, the left hand side of Eq. (C.2) is given in terms of the lower-dimensional fields by

$$
\begin{aligned}
\hat{\nabla}^{\mu} \hat{\xi}_{(b)}^{z}+\hat{\nabla}^{z} \hat{\xi}_{(b)}^{\mu} & =e^{-2 \alpha \varphi} g^{\mu \rho}\left(\mathcal{L}_{\xi} \mathcal{A}_{\rho}-\mathcal{A}^{\sigma} \mathcal{L}_{\xi} g_{\rho \sigma}\right)+\mathcal{A}^{\mu} \mathcal{L}_{\xi} e^{-2 \alpha \varphi} \\
& =0=\mathcal{L}_{\xi} \hat{g}^{\mu z}
\end{aligned}
$$

Obviously, the $(\mu, z)$ components of Eq. (C.2) also holds.

When $\hat{\mu}=z$ and $\hat{\nu}=z$, the left hand side of Eq. (C.2) is presented by

$$
\begin{aligned}
2 \hat{\nabla}^{z} \hat{\xi}_{(b)}^{z} & =-\mathcal{L}_{\xi}\left(e^{-2 \alpha \varphi} \mathcal{A}_{\mu} \mathcal{A}^{\mu}+e^{-2 \beta \varphi}\right) \\
& =0=-\mathcal{L}_{\xi} \hat{g}^{z z}
\end{aligned}
$$

which means that the $(z, z)$-component obeys Eq. (C.2). By a combination of Eqs. (C.4), (C.5) and (C.6), one sees that the $(D+1)$-dimensional Killing equation (C.2) indeed holds for all the components.

Finally, we show that the Lie derivative of the potential $\mathcal{L}_{\hat{\xi}} \hat{A}_{(n-1) \hat{\mu}_{1} \cdots \hat{\mu}_{(n-1)}}$ in $(D+1)$ dimensional spacetime along the Killing vector $\hat{\xi}^{\hat{\mu}}$ vanishes, that is,

$$
\mathcal{L}_{\hat{\xi}} \hat{A}_{(n-1) \hat{\mu}_{1} \cdots \hat{\mu}_{(n-1)}}=0,
$$

if the Lie derivative of the potentials in $D$-dimensional spacetime satisfies Eq. (5.7). In fact, according to the definition of the Lie derivative, one sees that

$$
\begin{aligned}
\mathcal{L}_{\hat{\xi}} \hat{A}_{(n-1) \mu_{1} \cdots \mu_{(n-1)}}= & \hat{\xi} \hat{\mu} \hat{\partial}_{\hat{\mu}} \hat{A}_{(n-1) \mu_{1} \cdots \mu_{(n-1)}} \\
& -\sum_{k=1}^{n-1}(-1)^{k} \hat{A}_{(n-1) \hat{\nu} \mu_{1} \cdots \mu_{(k-1)} \mu_{(k+1)} \cdots \mu_{(n-1)}} \hat{\partial}_{\hat{\mu}_{k}} \hat{\xi}^{\hat{\nu}} \\
= & \xi^{\mu} \partial_{\mu} A_{(n-1) \mu_{1} \cdots \mu_{(n-1)}} \\
& -\sum_{k=1}^{n-1}(-1)^{k} A_{(n-1) \nu \mu_{1} \cdots \mu_{(k-1)} \mu_{(k+1)} \cdots \mu_{(n-1)}} \partial_{\mu_{k}} \xi^{\nu} \\
= & \mathcal{L}_{\xi} A_{(n-1) \mu_{1} \cdots \mu_{(n-1)}}=0 .
\end{aligned}
$$

On the other hand, in the same manner, one gets

$$
\mathcal{L}_{\hat{\xi}} \hat{A}_{(n-1) \mu_{1} \cdots \mu_{(n-2)} z}=\mathcal{L}_{\xi} A_{(n-2) \mu_{1} \cdots \mu_{(n-2)}}=0 .
$$




\section{The Kaluza-Klein reduction of the Lagrangian with $p$-form potentials}

In this appendix, according to the Kaluza-Klein theory, we perform a dimensional reduction to the general Lagrangian including multiple $p$-form potentials and scalar fields from $(D+1)$ dimensions to $D$ dimensions on the circle $S^{1}$. As before, the $(D+1)$-dimensional spacetime is endowed with the coordinate system $\hat{x}^{\hat{\mu}}=\left(x^{\mu}, z\right)$, and the $(D+1)$-dimensional metric ansatz is still supposed to take the form given in Eq. (4.2).

As a warm-up, we prove that the contraction between the $(D+1)$-dimensional totally antisymmetric $n$-rank tensors $\hat{\Phi}_{(n)}^{\hat{\mu}_{1} \cdots \hat{\mu}_{n}}$ and $\hat{\Psi}_{\hat{\mu}_{1} \cdots \hat{\mu}_{n}}^{(n)}$ can be expressed as

$$
\begin{aligned}
\hat{\Phi}_{(n)}^{\hat{\mu}_{1} \cdots \hat{\mu}_{n}} \hat{\Psi}_{\hat{\mu}_{1} \cdots \hat{\mu}_{n}}^{(n)}= & \hat{g}^{\mu_{2} \nu_{2}} \cdots \hat{g}^{\mu_{n} \nu_{n}}\left(\hat{g}^{\mu_{1} \nu_{1}} \hat{\Phi}_{\nu_{1} \cdots \nu_{n}}^{(n)} \hat{\Psi}_{\mu_{1} \cdots \mu_{n}}^{(n)}+n \hat{g}^{z z} \hat{\Phi}_{z \nu_{2} \cdots \nu_{n}}^{(n)} \hat{\Psi}_{z \mu_{2} \cdots \mu_{n}}^{(n)}\right. \\
& \left.+n \hat{g}^{\mu_{1} z} \hat{\Phi}_{z \nu_{2} \cdots \nu_{n}}^{(n)} \hat{\Psi}_{\mu_{1} \cdots \mu_{n}}^{(n)}+n \hat{g}^{z \nu_{1}} \hat{\Phi}_{\nu_{1} \cdots \nu_{n}}^{(n)} \hat{\Psi}_{z \mu_{2} \cdots \mu_{n}}^{(n)}\right) \\
& +\frac{n(n-1)}{2} \hat{g}^{\mu_{1} z} \hat{g}^{z \nu_{2}} \hat{g}^{\mu_{3} \nu_{3}} \cdots \hat{g}^{\mu_{n} \nu_{n}} \hat{\Phi}_{z \nu_{2} \cdots \nu_{n}}^{(n)} \hat{\Psi}_{\mu_{1} z \mu_{3} \cdots \mu_{n}}^{(n)} \\
& +\frac{n(n-1)}{2} \hat{g}^{z \nu_{1}} \hat{g}^{\mu_{2} z} \hat{g}^{\mu_{3} \nu_{3}} \cdots \hat{g}^{\mu_{n} \nu_{n}} \hat{\Phi}_{\nu_{1} z \nu_{3} \cdots \nu_{n}}^{(n)} \hat{\Psi}_{z \mu_{2} \cdots \mu_{n}}^{(n)}
\end{aligned}
$$

Moreover, if

$$
\begin{array}{ll}
\hat{\Phi}_{(n) \mu_{1} \cdots \mu_{n}}=\Phi_{(n) \mu_{1} \cdots \mu_{n}}, & \hat{\Phi}_{(n) \mu_{1} \cdots \mu_{(n-1)} z}=\Phi_{(n-1) \mu_{1} \cdots \mu_{(n-1)}}, \\
\hat{\Psi}_{(n) \mu_{1} \cdots \mu_{n}}=\Psi_{(n) \mu_{1} \cdots \mu_{n}}, & \hat{\Psi}_{(n) \mu_{1} \cdots \mu_{(n-1)} z}=\Psi_{(n-1) \mu_{1} \cdots \mu_{(n-1)}},
\end{array}
$$

where $\Phi_{(n)}, \Phi_{(n-1)}, \Psi_{(n)}$ and $\Psi_{(n-1)}$ are totally antisymmetric tensors in $D$-dimensional spacetime, Eq. (D.1) can be simplified as

$$
\begin{aligned}
\hat{\Phi}_{(n)}^{\hat{\mu}_{1} \cdots \hat{\mu}_{n}} \hat{\Psi}_{\hat{\mu}_{1} \cdots \hat{\mu}_{n}}^{(n)}= & e^{-2 n \alpha \varphi}\left(\Phi_{(n)}^{\mu_{1} \cdots \mu_{n}}-n \Phi_{(n-1)}^{\left[\mu_{1} \cdots \mu_{(n-1)}\right.} \mathcal{A}^{\left.\mu_{n}\right]}\right) \\
& \times\left(\Psi_{\mu_{1} \cdots \mu_{n}}^{(n)}-n \Psi_{\left[\mu_{1} \cdots \mu_{(n-1)}\right.}^{(n-1)} \mathcal{A}_{\left.\mu_{n}\right]}\right) \\
& +n e^{2(D-n-1) \alpha \varphi} \Phi_{(n-1)}^{\mu_{1} \cdots \mu_{(n-1)}} \Psi_{\mu_{1} \cdots \mu_{(n-1)}}^{(n-1)}
\end{aligned}
$$

Now we move on to consider the Kaluza-Klein reduction of the $(D+1)$-dimensional Lagrangian for $p$-form potentials $\hat{A}_{(p)}$ and scalar fields $\phi^{(k)}(k=1, \cdots, m)$, which is postulated to take the general form

$$
\hat{\mathcal{L}}_{\hat{M}}=\sqrt{-\hat{g}} W\left(\phi^{(k)}\right) \hat{H}_{(N)}^{\hat{\mu}_{1} \cdots \hat{\mu}_{N}} \hat{Y}_{(N) \hat{\mu}_{1} \cdots \hat{\mu}_{N}}
$$


where the two totally antisymmetric $N$-rank tensors $\hat{H}_{(N)}$ and $\hat{Y}_{(N)}$ in $(D+1)$-dimensional spacetime are read off as

$$
\begin{aligned}
\hat{H}_{(N) \hat{\mu}_{1} \cdots \hat{\mu}_{N}} & =\left(\hat{F}_{\left(q_{1}\right)} \cdots \hat{F}_{\left(q_{t}\right)} \hat{A}_{\left(p_{1}\right)} \cdots \hat{A}_{\left(p_{s}\right)}\right)_{\left[\hat{\mu}_{1} \cdots \hat{\mu}_{N}\right]}, \\
\hat{Y}_{(N) \hat{\mu}_{1} \cdots \hat{\mu}_{N}} & =\left(\hat{F}_{\left(\tilde{q}_{1}\right)} \cdots \hat{F}_{\left(\tilde{q}_{j}\right)} \hat{A}_{\left(\tilde{p}_{1}\right)} \cdots \hat{A}_{\left(\tilde{p}_{i}\right)}\right)_{\left[\hat{\mu}_{1} \cdots \hat{\mu}_{N}\right]},
\end{aligned}
$$

taking the similar structures as the ones given in Eq. (3.5). In Eq. (D.5), all the potentials are defined by

$$
\begin{gathered}
\hat{A}_{\left(p_{c}\right)}=A_{\left(p_{c}\right)}+A_{\left(p_{c}-1\right)} \wedge d z, \quad 1 \leq c \leq s, \\
\hat{A}_{\left(\tilde{p}_{c}\right)}=A_{\left(\tilde{p}_{c}\right)}+A_{\left(\tilde{p}_{c}-1\right)} \wedge d z, \quad 1 \leq c \leq i, \\
\hat{A}_{\left(q_{c}-1\right)}=A_{\left(q_{c}-1\right)}+A_{\left(q_{c}-2\right)} \wedge d z, \quad 1 \leq c \leq t, \\
\hat{A}_{\left(\tilde{q}_{c}-1\right)}=A_{\left(\tilde{q}_{c}-1\right)}+A_{\left(\tilde{q}_{c}-2\right)} \wedge d z, \quad 1 \leq c \leq j,
\end{gathered}
$$

where all the quantities $A_{(p)}\left(p=p_{c}, p_{c}-1, \cdots, \tilde{q}_{c}-2\right)$ are the $D$-dimensional potentials, which only depend on the $x^{\mu}$ coordinates. As a consequence, the components of the $(D+1)$ dimensional field strengths $\hat{F}_{\left(q_{c}\right)}$ and $\hat{F}_{\left(\tilde{q}_{c}\right)}$, defined through $\hat{F}_{\left(q_{c}\right)}=d \hat{A}_{\left(q_{c}\right)}$ and $\hat{F}_{\left(\tilde{q}_{c}\right)}=d \hat{A}_{\left(\tilde{q}_{c}\right)}$ respectively, are expressed as

$$
\begin{aligned}
& \hat{F}_{\mu_{1} \cdots \mu_{q_{c}}}^{\left(q_{c}\right)}=F_{\mu_{1} \cdots \mu_{q_{c}}}^{\left(q_{c}\right)}, \quad \hat{F}_{\mu_{1} \cdots \mu_{\left(q_{c}-1\right)}}^{\left(q_{c}\right)}=F_{\mu_{1} \cdots \mu_{\left(q_{c}-1\right)}}^{\left(q_{c}-1\right)}, \\
& \hat{F}_{\mu_{1} \cdots \mu_{\tilde{q}_{c}}}^{\left(\tilde{q}_{c}\right)}=F_{\mu_{1} \cdots \mu_{\tilde{q}_{c}}}^{\left(\tilde{q}_{c}\right)}, \quad \hat{F}_{\mu_{1} \cdots \mu_{\left(\tilde{q}_{c}-1\right)} z}^{\left(\tilde{q}_{c}\right)}=F_{\mu_{1} \cdots \mu_{\left(\tilde{q}_{c}-1\right)}^{\left(\tilde{q}_{c}-1\right)} .} .
\end{aligned}
$$

In the above equation, the $l$-form $F_{(l)}=d A_{(l-1)}\left(l=q_{c}, q_{c}-1, \tilde{q}_{c}, \tilde{q}_{c}-1\right)$ are $D$-dimensional field strengths. Obviously, all the field strengths are independent of the $z$ coordinate. In addition, it is supposed that all the scalar fields $\phi^{(k)}$ do not rely on the $z$ variable, yielding that the function $W\left(\phi^{(k)}\right)$ does not depend on the $z$ coordinate too.

As in Eq. (D.2), we further introduce the following $D$-dimensional totally antisymmetric tensors, which are

$$
\begin{gathered}
H_{(N) \mu_{1} \cdots \mu_{N}}=\hat{H}_{(N) \mu_{1} \cdots \mu_{N}}, \quad H_{(N-1) \mu_{1} \cdots \mu_{(N-1)}}=\hat{H}_{(N) \mu_{1} \cdots \mu_{(N-1)} z} \\
Y_{(N) \mu_{1} \cdots \mu_{N}}=\hat{Y}_{(N) \mu_{1} \cdots \mu_{N}}, \quad Y_{(N-1) \mu_{1} \cdots \mu_{(N-1)}}=\hat{Y}_{(N) \mu_{1} \cdots \mu_{(N-1)} z},
\end{gathered}
$$

respectively. On basis of the $(D+1)$-dimensional metric ansatz (4.2), by performing KaluzaKlein reduction on a circle, together with help of Eq. (D.3), the higher-dimensional La- 
grangian $\hat{\mathcal{L}}_{\hat{M}}$ is rewritten in terms of the lower-dimensional fields as

$$
\begin{aligned}
\hat{\mathcal{L}}_{\hat{M}}= & \tilde{\mathcal{L}}_{M} \\
= & \sqrt{-g} W\left[e^{-2(N-1) \alpha \varphi}\left(H_{(N)}^{\mu_{1} \cdots \mu_{N}}-N H_{(N-1)}^{\left[\mu_{1} \cdots \mu_{(N-1)}\right.} \mathcal{A}^{\left.\mu_{N}\right]}\right)\right. \\
& \times\left(Y_{\mu_{1} \cdots \mu_{N}}^{(N)}-N Y_{\left[\mu_{1} \cdots \mu_{(N-1)}\right.}^{(N-1)} \mathcal{A}_{\left.\mu_{N}\right]}\right) \\
& \left.+N e^{2(D-N) \alpha \varphi} H_{(N-1)}^{\mu_{1} \cdots \mu_{(N-1)}} Y_{\mu_{1} \cdots \mu_{(N-1)}}^{(N-1)}\right] .
\end{aligned}
$$

\section{E The $\hat{K}_{(\hat{F})}^{\mu \nu}$ potential in terms of the D-dimensional fields}

To get the exact relationship of the off-shell ADT potentials in higher and lower dimensions, in this appendix, detailed calculations are presented to derive the expression for the $(\mu, \nu)$ component of the $(D+1)$-dimensional off-shell Noether potential $\hat{K}_{(\hat{F})}^{\hat{\mu} \hat{\nu}}\left(\hat{\xi}_{(b)}\right)$, defined through Eq. (5.12), in terms of all the $D$-dimensional fields, where the $\hat{\xi}_{(b)}^{\mu}$ vector, given in Eq. (C.1), has been proved to be the $(D+1)$-dimensional Killing vector.

For convenience, four tensors $\hat{P}_{(i)}^{\mu \nu}(i=1,2,3)$ and $\hat{P}_{(b)}^{\mu \nu}$ are introduced to express the $(\mu, \nu)$-component of the $(D+1)$-dimensional off-shell Noether potential $\hat{K}_{(\hat{F})}^{\hat{\mu} \hat{\nu}}$, appearing in Eq. (5.12), as the following form

$$
\hat{K}_{(\hat{F})}^{\mu \nu}\left(\hat{\xi}_{(b)}\right)=-2 n(n-1) \gamma\left[\left(\hat{P}_{(1)}^{\mu \nu}+\hat{P}_{(2)}^{\mu \nu}+\hat{P}_{(3)}^{\mu \nu}\right)+b \hat{P}_{(b)}^{\mu \nu}\right]
$$

where the quantities $\hat{P}_{(i)}^{\mu \nu}(i=1,2,3)$ are given by

$$
\begin{aligned}
& \hat{P}_{(1)}^{\mu \nu}=\hat{g}^{\mu \alpha} \hat{g}^{\nu \beta} \hat{F}_{(n) \alpha \beta} \hat{\mu}_{1} \cdots \hat{\mu}_{(n-2)} \xi^{\sigma} \hat{A}_{(n-1) \sigma \hat{\mu}_{1} \cdots \hat{\mu}_{(n-2)},}, \\
& \hat{P}_{(2)}^{\mu \nu}=\hat{g}^{\mu \alpha} \hat{g}^{\nu z} \hat{F}_{(n) \alpha z} \hat{\mu}_{1} \cdots \hat{\mu}_{(n-2)} \xi^{\sigma} \hat{A}_{(n-1) \sigma \hat{\mu}_{1} \cdots \hat{\mu}_{(n-2)}}, \\
& \hat{P}_{(3)}^{\mu \nu}=\hat{g}^{\mu z} \hat{g}^{\nu \beta} \hat{F}_{(n) z \beta} \hat{\mu}_{1} \cdots \hat{\mu}_{(n-2)} \xi^{\sigma} \hat{A}_{(n-1) \sigma \hat{\mu}_{1} \cdots \hat{\mu}_{(n-2)}},
\end{aligned}
$$

and the quantity $\hat{P}_{(b)}^{\mu \nu}$ is read off as

$$
\begin{aligned}
\hat{P}_{(b)}^{\mu \nu}= & \left(\hat{g}^{\mu \alpha} \hat{g}^{\nu \beta} \hat{F}_{(n) \alpha \beta} \hat{\mu}_{1} \cdots \hat{\mu}_{(n-2)}+\hat{g}^{\mu \alpha} \hat{g}^{\nu z} \hat{F}_{(n) \alpha z} \hat{\mu}_{1} \cdots \hat{\mu}_{(n-2)}\right. \\
& \left.+\hat{g}^{\mu z} \hat{g}^{\nu \beta} \hat{F}_{(n) z \beta} \hat{\mu}_{1} \cdots \hat{\mu}_{(n-2)}\right) \hat{A}_{(n-1) z \hat{\mu}_{1} \cdots \hat{\mu}_{(n-2)}} \\
= & (-1)^{n} e^{-2 n \alpha \varphi} \tilde{F}_{(n)}^{\mu \nu \mu_{1} \cdots \mu_{(n-2)}} A_{\mu_{1} \cdots \mu_{(n-2)}}^{(n-2)} .
\end{aligned}
$$

To get the last equality in the above equation, Eq. (D.1) has been used. By utilizing Eq. 
(D.1) once again, we obtain that

$$
\begin{aligned}
\hat{P}_{(1)}^{\mu \nu}= & e^{-2 n \alpha \varphi} \xi^{\sigma}\left[A_{\sigma \mu_{1} \cdots \mu_{(n-2)}}^{(n-1)}\left(F_{(n)}^{\mu \nu \mu_{1} \cdots \mu_{(n-2)}}-(n-2) F_{(n-1)}^{\mu \nu \mu_{1} \cdots \mu_{(n-3)}} \mathcal{A}^{\mu_{(n-2)}}\right)\right. \\
& -(n-2) A_{\sigma \mu_{1} \cdots \mu_{(n-3)}}^{(n-2)}\left((n-3) F_{(n-1)}^{\mu \nu \mu_{1} \cdots \mu_{(n-4)} \rho} \mathcal{A}_{\rho} \mathcal{A}^{\mu_{(n-3)}}\right. \\
& \left.\left.+F_{(n)}^{\mu \nu \mu_{1} \cdots \mu_{(n-3)} \rho} \mathcal{A}_{\rho}-\mathcal{A}^{2} F_{(n-1)}^{\mu \nu \mu_{1} \cdots \mu_{(n-3)}}\right)\right] \\
& +(n-2) e^{2(D-n-1) \alpha \varphi} F_{(n-1)}^{\mu \nu \mu_{1} \cdots \mu_{(n-3)}} \xi^{\sigma} A_{\sigma \mu_{1} \cdots \mu_{(n-3)}}^{(n-2)},
\end{aligned}
$$

and the quantities $\hat{P}_{(2)}^{\mu \nu}$ and $\hat{P}_{(3)}^{\mu \nu}$ take the following values

$$
\begin{aligned}
& \hat{P}_{(2)}^{\mu \nu}=-e^{-2 n \alpha \varphi} \xi^{\sigma} \mathcal{A}^{\nu} F_{(n-1)}^{\mu \mu_{1} \cdots \mu_{(n-2)}}\left(A_{\mu_{1} \cdots \mu_{(n-2)} \sigma}^{(n-1)}+(n-2) A_{\mu_{1} \cdots \mu_{(n-3)} \sigma}^{(n-2)} \mathcal{A}_{\mu_{(n-2)}}\right), \\
& \hat{P}_{(3)}^{\mu \nu}=-\hat{P}_{(2)}^{\mu \nu}(\mu \leftrightarrow \nu) .
\end{aligned}
$$

Through a combination of Eqs. (E.3), (E.4) and (E.5), the off-shell Noether potential $\hat{K}_{(\hat{F})}^{\mu \nu}\left(\hat{\xi}_{(b)}\right)$ can be rewritten in terms of the $D$-dimensional fields as the form

$$
\hat{K}_{(\hat{F})}^{\mu \nu}\left(\hat{\xi}_{(b)}\right)=e^{-2 \alpha \varphi} K_{(F)}^{\mu \nu}(\xi)-2 b n(n-1) \gamma e^{-2 n \alpha \varphi} \tilde{F}_{(n)}^{\mu \mu_{1} \cdots \mu_{(n-2)} \nu} A_{\mu_{1} \cdots \mu_{(n-2)}}^{(n-2)} .
$$

Here the $n$-form field strength $\tilde{F}_{(n)}$ is given by Eq. (5.6).

\section{References}

[1] L. F. Abbott and S. Deser, Stability of gravity with a cosmological constant, Nucl. Phys. $B 195$ (1982) 76; L. F. Abbott and S. Deser, Charge definition in non-abelian gauge theories, Phys. Lett. B 116 (1982) 259.

[2] S. Deser and B. Tekin, Gravitational Energy in Quadratic Curvature Gravities, Phys. Rev. Lett. 89 (2002) 101101 arXiv:hep-th/0205318; S. Deser and B. Tekin, Energy in Generic Higher Curvature Gravity Theories, Phys. Rev. D 67 (2003) 084009 arXiv:hep-th/0212292.

[3] S. Deser and B. Tekin, New energy definition for higher curvature gravities, Phys. Rev. D 75 (2007) 084032 [arXiv:gr-qc/0701140].

[4] S. Deser and B. Tekin, Energy in topologically massive gravity, Class. Quant. Grav. 20 (2003) L259 arXiv:gr-qc/0307073. 
[5] A. Bouchareb and G. Clement, Black-hole mass and angular momentum in topologically massive gravity, Class. Quantum Grav. 24 (2007) 5581 [arXiv:0706.0263].

[6] K. Ait Moussa, G. Clement, H. Guennoune and C. Leygnac, Three-dimensional ChernSimons black holes, Phys. Rev. D 78 (2008) 064065 arXiv:0807.4241.

[7] S. Nam, J.D. Park and S.H. Yi, Mass and Angular momentum of Black Holes in New Massive Gravity, Phys. Rev. D 82 (2010) 124049 arXiv:1009.1962.

[8] K. Ait Moussa, G. Clement and H. Guennoune, Chern-Simons dilaton black holes in 2+1 dimensions, Class. Quantum Grav. 33 (2016) 065008 arXiv:1510.07152.

[9] W. Kim, S. Kulkarni, and S.H. Yi, Quasi-Local Conserved Charges in Covariant Theory of Gravity, Phys. Rev. Lett. 111 (2013) 081101 [arXiv:1306.2138].

[10] S. Hyun, J. Jeong, S.A Park and S.H. Yi, Quasi-local conserved charges and holography, Phys. Rev. D 90 (2014) 104016 arXiv:1406.7101.

[11] J.J. Peng, Conserved charges of black holes in Weyl and Einstein-Gauss-Bonnet gravities, Eur. Phys. J. C $\mathbf{7 4}$ (2014) 3156 [arXiv:1407.4875].

[12] M.R. Setare and H. Adami, Quasi-local conserved charges in the Einstein-Maxwell theory, Class. Quantum Grav. 34 (2017) 105008 arXiv:1609.06168.

[13] J.J. Peng, W.C. Xiang and S.H. Cai, Abbott-Deser-Tekin Charge of Dilaton Black Holes with Squashed Horizons, Chin. Phys. Lett. 33 (2016) 080401.

[14] J.J. Peng, Mass and angular momentum of black holes in low-energy heterotic string theory, Int. J. Mod. Phys. A 31 (2016) 1650060 [arXiv:1604.06619].

[15] S.L. Li, H. Lu and H. Wei, Dyonic (A)dS Black Holes in Einstein-Born-Infeld Theory in Diverse Dimensions, JHEP 1607 (2016) 004 [arXiv:1606.02733].

[16] S.Q. Wu and S.L. Li, Thermodynamics of static dyonic AdS black holes in the $\omega$ deformed Kaluza-Klein gauged supergravity theory, Phys. Lett. B $\mathbf{7 4 6}$ (2015) 276 arXiv:1505.00117.

[17] J.J. Peng, Off-shell Noether current and conserved charge in Horndeski theory, Phys. Lett. B 752 (2016) 191 arXiv:1511.06516]. 
[18] S. Nam and J.D. Park, Mass and Angular Momentum of Black Holes in Three Dimensional Gravity Theories with First Order Formalism, arXiv:1606.05997.

[19] S. Hyun, S.A Park and S.H. Yi, Quasi-local charges and asymptotic symmetry generators, JHEP 06 (2014) 151 [arXiv:1403.2196].

[20] M.R. Setare and H. Adami, Lorentz-diffeomorphism Conserved Charges and Virasoro Algebra in Chern-Simons-Like Theories of Gravity, Nucl. Phys. B 909 (2016) 345 [arXiv:1511.01070].

[21] W. Kim, S. Kulkarni and S.H. Yi, Quasilocal Conserved Charges with a Gravitational Chern-Simons Term, Phys. Rev. D 88 (2013) 124004 [arXiv:1310.1739].

[22] T. Padmanabhan, Entropy density of spacetime and thermodynamic interpretation of field equations of gravity in any diffeomorphism invariant theory, arXiv:0903.1254.

[23] T. Padmanabhan, Thermodynamical Aspects of Gravity: New insights, Rept. Prog. Phys. 73 (2010) 046901 [arXiv:0911.5004].

[24] J. Lee and R. M. Wald, Local symmetries and constraints, J. Math. Phys. 31 (1990) 725.

[25] V. Iyer and R.M. Wald, Some properties of the Noether charge and a proposal for dynamical black hole entropy, Phys. Rev. D 50 (1994) 846 arXiv:gr-qc/9403028.

[26] V. Iyer and R.M. Wald, A Comparison of Noether charge and Euclidean methods for computing the entropy of stationary black holes, Phys. Rev. D 52 (1995) 4430 arXiv:gr-qc/9503052.

[27] G. Barnich and F. Brandt, Covariant theory of asymptotic symmetries, conservation laws and central charges, Nucl. Phys. B 633 (2002) 3 [arXiv:hep-th/0111246].

[28] G. Barnich, Boundary charges in gauge theories: using Stokes theorem in the bulk, Class. Quantum Grav. 20 (2003) 3685 [arXiv:hep-th/0301039].

[29] G. Barnich and G. Compere, Generalized Smarr relation for Kerr AdS black holes from improved surface integrals, Phys. Rev. D 71 (2005) 044016 [Erratum ibid 73 (2006) 029904] [arXiv:gr-qc/0412029]. 
[30] G. Barnich and G. Compere, Surface charge algebra in gauge theories and thermodynamic integrability, J. Math. Phys. 49 (2008) 042901 [arXiv:0708.2378].

[31] T. Liko, Isolated horizons, p-form matter fields, topology and the black-hole/string correspondence principle, Phys. Rev. D 79 (2009) 084038 [arXiv:0901.1121].

[32] K. Hajian, On Thermodynamics and Phase Space of Near Horizon Extremal Geometries, Ph.D thesis, (2015) [arXiv:1508.03494].

[33] K. Hajian and M.M. Sheikh-Jabbari, Solution Phase Space and Conserved Charges: A General Formulation for Charges Associated with Exact Symmetries, Phys. Rev. D 93 (2016) 044074 [arXiv:1512.05584].

[34] M. Ghodrati, K. Hajian and M.R. Setare, Revisiting Conserved Charges in Higher Curvature Gravitational Theories, [arXiv:1606.04353].

[35] C.N. Pope, Lectures on Kaluza-Klein Theory (unpublished notes), http://people.physics.tamu.edu/pope/ihplec.pdf.

[36] M. Rogatko, First law of black rings thermodynamics in higher dimensional dilaton gravity with $p+1$ strength forms, Phys. Rev. D 73 (2006) 024022 [arXiv:hepth/0601055]; M. Rogatko, Physical process version of the first law of thermodynamics for black holes in higher dimensional gravity, Phys. Rev. D 71 (2005) 104004 [arXiv:hep-th/0505047].

[37] G. Compere, Note on the First Law with p-form potentials, Phys. Rev. D 75 (2007) 124020 [arXiv:hep-th/0703004].

[38] D.D.K. Chow and G. Compere, Dyonic AdS black holes in maximal gauged supergravity, Phys. Rev. D 89 (2014) 065003 [arXiv:1311.1204].

[39] S.Q. Wu, General Nonextremal Rotating Charged AdS Black Holes in Five-dimensional $U(1)^{3}$ Gauged Supergravity: A Simple Construction Method, Phys. Lett. B 707 (2012) 286 [arXiv:1108.4159].

[40] S. Tomizawa and S. Mizoguchi, General Kaluza-Klein black holes with all six independent charges in five-dimensional minimal supergravity, Phys. Rev. D 87 (2013) 024027 [arXiv:1210.6723]; S. Mizoguchi and S. Tomizawa, New approach to solution generation 
using $S L(2, R)$-duality of a dimensionally reduced space in five-dimensional minimal supergravity and new black holes, Phys. Rev. D 84 (2011) 104009 [arXiv:1106.3165].

[41] S.Q. Wu, General nonextremal rotating charged Gödel black holes in minimal fivedimensional gauged supergravity, Phys. Rev. Lett. 100 (2008) 121301 [arXiv:0709.1749]; E. Gimon and A. Hashimoto, Black holes in Gödel universes and pp waves, Phys. Rev. Lett. 91 (2003) 021601 [arXiv:hep-th/0304181].

[42] Z.W. Chong, M. Cvetic, H. Lu and C.N. Pope, General non-extremal rotating black holes in minimal five-dimensional gauged supergravity, Phys. Rev. Lett. 95 (2005) 161301 [arXiv:hep-th/0506029].

[43] S.Q. Wu, General rotating charged Kaluza-Klein AdS black holes in higher dimensions, Phys. Rev. D 83 (2011) 121502 [arXiv:1108.4157].

[44] S. Deser and B. Tekin, Conformal Properties of Charges in Scalar-Tensor Gravities, Class. Quant. Grav. 23 (2006) 7479 arXiv:gr-qc/0609111.

[45] F. Larsen, Rotating Kaluza-Klein black holes, Nucl. Phys. B 575 (2000) 211 arXiv:hep-th/9909102.

[46] T. Azeyanagi, N. Ogawa and S. Terashima, Holographic Duals of Kaluza-Klein Black Holes, JHEP 0904 (2009) 061 [arXiv:0811.4177]. 